Review

\title{
The Impact of the COVID-19 Pandemic on Burnout, Compassion Fatigue, and Compassion Satisfaction in Healthcare Personnel: A Systematic Review of the Literature Published during the First Year of the Pandemic
}

\author{
Cristina Lluch ${ }^{1}$, Laura Galiana ${ }^{1} \mathbb{D}$, Pablo Doménech ${ }^{2}$ and Noemí Sansó ${ }^{3,4, * \mathbb{D}}$ \\ 1 Department of Methodology for the Behavioral Sciences, University of Valencia, 46010 Valencia, Spain; \\ cris9@alumni.uv.es (C.L.); laura.galiana@uv.es (L.G.) \\ 2 Faculty of Education, Valencian International University, 46002 Valencia, Spain; \\ pablo.domenech@campusviu.es \\ 3 Department of Nursing and Physiotherapy, University of Balearic Islands, 07122 Palma, Spain \\ 4 Balearic Islands Health Research Institute (IDISBA), 07120 Palma, Spain \\ * Correspondence: noemi.sanso@uib.es
}

\section{check for}

updates

Citation: Lluch, C.; Galiana, L.;

Doménech, P.; Sansó, N. The Impact of the COVID-19 Pandemic on Burnout, Compassion Fatigue, and Compassion Satisfaction in Healthcare Personnel: A Systematic Review of the Literature Published during the First Year of the Pandemic. Healthcare 2022, 10, 364. https:// doi.org/10.3390/healthcare10020364

Academic Editor: Alyx Taylor

Received: 20 January 2022

Accepted: 10 February 2022

Published: 13 February 2022

Publisher's Note: MDPI stays neutral with regard to jurisdictional claims in published maps and institutional affiliations.

Copyright: (C) 2022 by the authors. Licensee MDPI, Basel, Switzerland. This article is an open access article distributed under the terms and conditions of the Creative Commons Attribution (CC BY) license (https:/ / creativecommons.org/licenses/by/ $4.0 /)$.

\begin{abstract}
This literature review aimed to determine the level of burnout, compassion fatigue, and compassion satisfaction, as well as their associated risks and protective factors, in healthcare professionals during the first year of the COVID-19 pandemic. We reviewed 2858 records obtained from the CINAHL, Cochrane Library, Embase, PsycINFO, PubMed, and Web of Science databases, and finally included 76 in this review. The main results we found showed an increase in the rate of burnout, dimensions of emotional exhaustion, depersonalization, and compassion fatigue; a reduction in personal accomplishment; and levels of compassion satisfaction similar to those before the pandemic. The main risk factors associated with burnout were anxiety, depression, and insomnia, along with some sociodemographic variables such as being a woman or a nurse or working directly with COVID-19 patients. Comparable results were found for compassion fatigue, but information regarding compassion satisfaction was lacking. The main protective factors were resilience and social support.
\end{abstract}

Keywords: COVID-19; burnout; compassion fatigue; compassion satisfaction; health personnel

\section{Introduction}

Because healthcare professionals are especially exposed at the frontline of the COVID19 pandemic, their quality of life has been put at great risk. Among several potentially harmful factors for the health of professionals, some authors have highlighted the lack of access to adequate protective equipment [1], exhaustion resulting from wearing personal protective equipment throughout the working day, the feeling of having inadequate support [2,3], long working hours and unexpected changes in the type of work [4], concern about trapping or infecting their relatives [5], abandoning their homes to avoid infecting their families [6], lack of access to updated information on constantly changing patterns of action [3,7], uncertainty about disease containment [1], and concerns about seeing patients die [5].

Thus, it seems clear that health professionals are under extreme psychological pressure and, consequently, are at risk of developing several psychological symptoms and mental health disorders [3]. For example, a recent review that included data from more than 7000 professionals [4] found that the prevalence of PTSD symptoms and anxiety and depression ranged from $9.6 \%$ to $51 \%$ and $20 \%$ to $75 \%$, respectively. High levels of stress and somatic symptoms were also reported in Italian health professionals in the study by Barello et al. [8]. Furthermore, a study by Kotera et al. [9] in Japan, found that physicians 
had more mental health disorders, felt more alone, and had less hope and self-compassion compared to the general population.

Therefore, it is not surprising that the COVID-19 pandemic has worsened the quality of life of professionals, aggravating pre-existing problems such as burnout. Burnout, or professional burnout, is a syndrome that occurs in service sector workers subjected to stressful situations [10], and can be defined as the "result of chronic stress in the workplace that has not been successfully managed" [11]. The academic literature from the past few decades has revealed that health professionals are especially vulnerable to burnout because their work context is characterized by high-risk decisions, dealing with the public, and expectations of compassion and sensitivity [12]. However, more and more academics and clinicians have pointed out that burnout alone is insufficient to explain the emotional problems presented by practitioners in general healthcare contexts $[13,14]$ or in the field of palliative care, in particular $[15,16]$. In this sense, a large body of recent evidence now suggests that many healthcare professionals are suffering from compassion fatigue $[17,18]$.

The concept of compassion fatigue was first introduced by Joinson [19] to characterize a state of reduced capacity for compassion as a consequence of exhaustion caused by contact with the suffering of others [20]. Witnessing the suffering of patients without being able to alleviate their discomfort has a high emotional toll on healthcare personnel [21]. The most widespread theoretical model for the study of compassion fatigue is currently the one developed by Beth Stamm [22,23], who defined it as the negative aspect of professional quality of life and divided it into two dimensions: (1) burnout (as previously explained), and (2) secondary trauma, vicarious trauma, or secondary traumatic stress, which refers to negative feelings driven by fear and trauma related to work [23]. This model also studies the opposite pole of compassion fatigue, that is, the positive aspects of professional life, or compassion satisfaction.

Compassion satisfaction occurs when exposure to traumatic and distress-related events produces satisfaction [24] derived from the pleasure of helping others [22] and providing a means to alleviate suffering [24]. Indeed, when helping people and changing their lives is managed appropriately, professionals and caregivers can feel pleasure and satisfaction rather than burnout or compassion fatigue [14]. Therefore, considering the definition of professional quality of life, it seems clear that the circumstances created by the COVID-19 pandemic are a clear threat to the mental health of professionals and may have affected their levels of burnout, compassion fatigue, and compassion satisfaction. In turn, these factors are key to achieving the adequate well-being of healthcare providers [25-28] and, in turn, can strongly affect the quality of care received by patients and their families [29].

Although several systematic reviews have recently been published on the impact of the COVID-19 pandemic on the mental health of healthcare professionals $[4,30,31]$, very few of them specifically included dimensions of professional quality of life such as burnout [32-35], only one considered indirect trauma [36] and, to the best of our knowledge, none reviewed the evidence on compassion satisfaction. In this context, the main objective of this current work was to understand the impact of the COVID-19 pandemic on burnout, compassion fatigue, and compassion satisfaction among health professionals by systematically reviewing the literature published during the first year of the COVID-19 pandemic. Specifically, we aimed to answer the following questions, all of them referring to the experience lived during the first year of the COVID-19 pandemic:

1. What levels of burnout, compassion fatigue, and compassion satisfaction have health professionals who worked during the COVID-19 pandemic experienced?

2. What variables (risk factors) were related to the COVID-19 pandemic having a greater negative impact on professional quality of life?

3. What variables (protective factors) corresponded to the COVID-19 pandemic having a lower negative impact on professional quality of life? 


\section{Materials and Methods}

To complete this work we conducted a systematic review of the scientific literature, following the Preferred Reporting Items for Systematic Review and Meta-analysis (PRISMA) guidelines [37] (see Online Supplement S1).

\subsection{Eligibility Criteria}

\subsubsection{Type of Participants}

Health professionals (physicians, nurses, nursing assistants, psychologists, etc.) who carried out their professional activities in the health system (such as primary healthcare centers, emergency departments, intensive care units, palliative care units, or COVID-19 services, among others) during the COVID-19 pandemic were considered in this work.

\subsubsection{Study Variables}

We considered studies that addressed burnout, compassion fatigue, and compassion satisfaction in health professionals who had cared for patients infected by COVID-19.

\subsubsection{Study Types}

We included quantitative studies (either cross-sectional or longitudinal) with primary data that addressed burnout, compassion fatigue, and compassion satisfaction in healthcare professionals during the COVID-19 pandemic. Studies published, or that were in press, from 1 January 2020 to 31 December 2020, were considered.

\subsubsection{Language}

Articles published in English or Spanish were included.

\subsubsection{Publication Date}

Articles published during 2020 (from 1 January 2020 to 31 December 2020) were considered.

\subsubsection{Exclusion Criteria}

The following types of work were excluded: studies that did not consider healthcare professionals; did not include our study variables (burnout, compassion fatigue, and/or compassion satisfaction); did not include quantitative primary data (i.e., single case studies, reviews, letters to the Editor, comments, qualitative studies, etc.); were not published in Spanish or English; were not published during the year 2020; and that included data from before the COVID-19 pandemic, even when the work met all the inclusion criteria.

\subsection{Data Sources and Search Strategy}

We searched the CINAHL, Cochrane Library, Embase, PsycINFO, PubMed, and Web of Science databases for relevant articles. Thus, we only used reliable, peer-reviewed databases, platforms, and sources with search tools that allowed us to access the study dates, and thereby, systematically identify studies. These databases included academic literature related to various health disciplines, including health psychology, and therefore represented reliable sources of expert research and information.

The keywords we used were:

- Pandemic or COVID-19 or SARS-CoV-2 or Coronavirus, as well as the synonyms for these terms included in the Medical Subject Headings (MeSH) database; and

- Burnout or compassion fatigue or stress disorders or compassion satisfaction, as well as the synonyms for these terms included in the MeSH; and

- Health personnel or nursing staff or nurses or physicians or psychology, as well as the synonyms for these terms included in the MeSH.

A list of the terms found in the $\mathrm{MeSH}$, together with the equation we used in the final search, is provided in Online Supplement S2. 
Regarding the review procedure, first we entered the search equation into each of the databases, filtering them by publication date (1 January 2020 to 31 December 2020) to narrow the results based on the eligibility criteria. Second, the eligible papers were identified based on their title and keywords as well as whether they met the inclusion criteria. Third, we read the abstracts, reserving any studies we believed met the inclusion criteria. Finally, the full texts of these articles were obtained and read. After this reading, we chose the final records for inclusion and performed the data synthesis.

\subsection{Data Extraction and Synthesis}

The data from the publications obtained in the search strategy were extracted into an Excel template that was modified according to the studies we reviewed. The metadata included the author(s), year of publication, country of study, main study objective, design, sample size, types of participating professionals, distribution by gender, mean age, other sample characteristics, assessment instruments, metrics used for each variable, descriptive and inferential results relative to the prevalence, data collection date, risk factors and protective variables for burnout, compassion fatigue, and compassion satisfaction. Specifically:

1. Means and standard or median deviations and interquartile ranges (for quantitative data), frequencies and percentages (for categorical data) of the prevalence data for burnout, compassion fatigue, and compassion satisfaction.

2. To study the risk factors and protective variables of burnout, compassion fatigue, and compassion satisfaction, chi-squared tests, contrast of means, and analysis of variance (for categorical variables), Pearson correlations, Spearman correlations, and simple and multiple regressions (for quantitative variables) were used.

\section{Results}

When applying the inclusion and exclusion criteria to the results from the six databases, the search equation produced 2856 records. As shown in Figure 1, these were reduced to 2498 records once the publication date was limited. We reviewed all these entries, first by title and then by abstract, leaving a total of 234 total records for full text review. Most of these were excluded because they did not meet one or more of the inclusion criteria (i.e., health professional participants, burnout, compassion fatigue, compassion satisfaction variables, etc.). After reading the full texts of all these entries, 76 records were retained for inclusion in this review. The main characteristics of these tests are summarized in Table 1. 


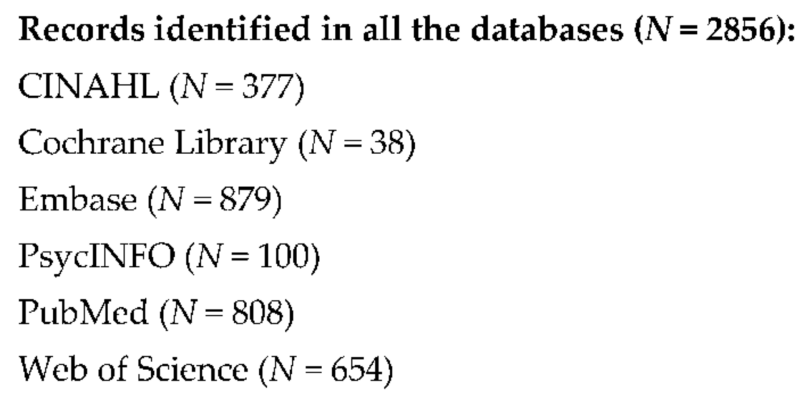

Number of records finally included in this review $(N=76)$

Figure 1. Article selection flow chart. 
Table 1. The main characteristics of the studies included in this systematic review.

Author and Year

Aims
Country

Sample

Result

Vicarious trauma (VTS): The mean level of

moderate vicarious trauma was $M=33.46$ $8.5 \%$. Vicarious trauma was greater in therapists who experienced more distress in response to what they had heard in session during the pandemic compared to previous sessions $(r=0.22, p<0.001)$. They felt more tired $(r=0.16, p<0.010)$, less competent $(r=0.21, p<0.001)$, and less confident $(r=0.15$ $(r=0.21, p<0.001)$, and less confident $(r=0.1$ $p<0.010$ ) in their therapy sessions compared to their pre-pandemic feelings. They also reported a deterioration in their therapeutic work: less emotional connection with their clients $(r=0.18, p<0.001)$, and a weaker therapeutic relationship $(r=0.16, p<0.010)$ post-pandemic.

Burnout (MBI-HSS): 28.18\% presented severe exhaustion, $31.82 \%$ severe depersonalization, and $89.09 \%$ a severe decline in personal accomplishment. The lowest personal accomplishment scores were correlated with dissatisfaction with patient cure rates $(p=0.040)$ and the death of a colleague or $(p=0.040)$ and the death of a colleague or family member from COVID-19 $(p=0.040)$. The highest scores for emotional exhaustion $(p<0.001)$ and depersonalization $(p=0.004)$ were associated with the need to buy persona protective equipment (PPE) with personal money, and harassment by patient families while working $(p<0.001$ for both). Emotional exhaustion was associated with the absence of PPE in the workplace $(p=0.040)$, a lack of public awareness of the disease $(p=0.010)$, insufficient public appreciation of the work insufficient public appreciation of the
undertaken by physicians during the undertaken by physicians during the
pandemic $(p=0.010)$, and dissatisfaction with pandemic $(p=0.010)$, and
their salaries $(p=0.020)$ 
Table 1. Cont.

Author and Year

Aebischer et al. (2020) [40]
To compare the physical and

pychological health of Swiss medica students involved in the COVID-19 response with that of their non-involved peers. To compare the health of those who work on the frontline with that of peers not on the frontline. To compare frontline medical students with frontline medical residents. $n=777$

Students involved in COVID-19: 296

Students not involved in COVID-19: 254 Medical residents: 227

Men: 250

Women: 527
Switzerland

Burnout (MBI), generalized anxiety (GAD-7), and depression (PHQ-9): Students involved in the response to COVID-19 reported a similar the response to COVID-19 reported a simil
proportion of COVID-19 symptoms or confirmed diagnoses $(p=0.810)$, but lower levels of anxiety $(p<0.001)$, depression $(p<0.001)$, and depersonalization $(p<0.001)$, compared to their non-involved peers. The health of frontline students did not differ significantly from those who were not frontline. Frontline students had lower levels of emotional exhaustion than frontline residents $(p<0.010)$

Burnout: In response to the specific indicator, "Are you feeling exhausted right now?",

participants responded with yes/no: yes: 52

Characterize the degree of burnout and specific characteristics related to the United States of America COVID-19 pandemic. $n=100$ interventional pain physicians Men: 81 ( $81 \%)$ Women: $19(19 \%)$

$(52 \%) ;$ in: $46(46 \%)$; unanswer. $2(2 \%)$. Most reported that COVID-19 had impacted their operation (main modifications: telemedicin and interruption of procedures [98\%]; a reduction in earnings [91\%]; and reduced spending due to uncertainty $[77 \%]$ ).

Secondary trauma (STSS) and anxiety and depression (PHQ-4): Health workers who worked with COVID-19 patients had the highest secondary trauma score

$(M=2.66 \pm 0.96)$ while the general population obtained the lowest score $(M=2.34 \pm 0.76)$.

$n=563$

Health workers: 251

To study the differences in secondary

Arpacioglu et al. (2020) [17] trauma experienced by frontline workers Turkey and by the general population.
Physicians: 12

Nurses: 93

General population (non-health

workers): 95

Men: $212(37.7 \%)$

Women: $351(62.3 \%)$
Anxiety/depression levels: health workers working with COVID-19 patients

$(M=2.25 \pm 0.79)$, those not working with COVID-19 patients $(M=2.01 \pm 0.70)$,

non-health workers $(M=1.82 \pm 0.59)$. Women presented higher compassion fatigue $(p=0.048)$, which was significantly higher in nurses $(p=0.004)$ and physicians $(p=0.022)$ compared to the general population. Anxiety was significantly higher in nurses compared to other health professionals $(p=0.038)$. 
Table 1. Cont.

Author and Year

Azoulay et al. (2020) [42

Barello et al. (2020a) [8]
Document the prevalence of mental

health outcomes in intensive care unit

outbreak.
Country $n=1001 \mathrm{ICU}$ staff

Men: 659

Women: 342
Sample

Burnout (MBI) and anxiety and depression (HADS): Factors independently associated with symptoms of severe burnout included age (hazard ratio $[\mathrm{HR}]=0.98 /$ year $[0.97-0.99]$ ) an the physicians' rating of the ethical climate $(\mathrm{HR}=0.76$ [0.69-0.82]). Factors associated with the prevalence of depression symptoms were female sex $(42.4 \%$ vs. $30.5 \%$; $p<0.001)$ younger age (44 (38-51)vs. 46 years (39-54), $p=0.004)$, being single ( $21.4 \%$ vs. $15.1 \%$, $p=0.030)$, living in a city with $>1$ million inhabitants $(63.6 \%$ vs. $49.5 \%, p<0.001)$, and inhabitants (63.6\% a city with $>1$ million, and greater religiosity (36 (5-67) vs. $0.21(0-59)$, $p=0.002)$. Factors that were independently associated with anxiety symptoms were female $\operatorname{sex}(\mathrm{HR}=1.85$ [1.33-2.55]), work in a university hospital $(\mathrm{HR}=0.58(0.42-0.80))$, life in a city with $>1$ million inhabitants $(\mathrm{HR}=1.40$ (1.01-1.94)), and the physicians' rating of the ethical climate (HR $=0.83(0.77-0.90)$ ).

Burnout (MBI): $41 \%$ showed elevated levels of emotional exhaustion and $27 \%$ showed elevated levels of depersonalization. The perceived levels of professional risk $r=0.360$ $(p<0.001)$, emotional demands $r=0.244$

To describe the burnout levels of a sample of Italian healthcare workers involved in the management of the COVID-19 pandemic. To explore the relationship between professional

\section{Italy}

$n=532$

burnout and psychosomatic symptoms,

and perceived work demands and work

resources.
Nurses: 327

Physicians: 106

Others: 99

Men: 133

Women: 399 
Table 1. Cont.

Author and Year Aims
To report work-related psychological pressure, emotional exhaustion, and somatic symptoms during the COVID-19 Italy outbreak among healthcare workers in Italy.
Country

Sample

$$
n=376
$$

Hospital services: 307

Rehabilitation centers: 6

Outpatient: 3

Private centers:

Others: 55

Not specified: 4

Men: 99 (26.3\%)

Women: 277 (73.7\%)

To analyze the psychological

consequences (secondary traumatic stress and fear of contagion) of

COVID-19 in workers in nursing home

for the elderly. To study the influence of

stressors (workload, social pressure from Spain

work, and contact with death and

suffering) and inadequate work

resources (lack of personnel, materials,

and PPE, and insufficient support from

coworkers and supervisors). $n=228$

Physicians: $7.4 \%$

Nurses: $19.3 \%$

Nursing assistants: $30.3 \%$

Geriatric assistants: $6.5 \%$

Social workers: $15.3 \%$

Psychologists: $7.9 \%$

Occupational therapists: $4.8 \%$

Physiotherapists: $2.6 \%$

Managers: $1.3 \%$

Men: $19.7 \%$

Women: $80.3 \%$

\section{Results}

Burnout (MBI): Mean emotional exhaustion score $M=22.7(12.1 \%)$; depersonalization $M=6.1(5.7 \%)$; personal accomplishment $M=37.5(7.6 \%)$. The results showed a strong effect of gender on emotional exhaustion $(F(1.312)=12.444 ; p<0.001 ; \eta p 2=0.038)$, with women showing higher levels than men $(M=24.05 \pm 11.57$ and $M=18.74 \pm 12.65$ respectively).

Secondary trauma (STSQ): Workers in contact with COVID-19 patients $(n=156) M=2.80$; workers not in contact with COVID-19 patients $(n=70) M=2.62$. Workload stressors were related to secondary traumatic stress $(r=0.40$ $p<0.01 ; r=0.47, p<0.01 ; r=0.45, p<0.01$, respectively). Only the lack of personnel and PPE were associated with secondary traumatic stress $(r=0.33, p<0.01)$. Secondary trauma stress $(r=0.33, p<0.01)$. Secondary traum
was related to fear of contagion $(r=0.38$, was related to fear of contagion $(r=0.38$,
$p<0.01)$. Professionals in contact with patient $p<0.01)$. Professionals in contact with patients higher levels of secondary traumatic stress than professionals working in nursing homes with no detected cases $(M=2.80>M=2.62$; $t=3,05, p<0.01, d=0.46)$.

Burnout, secondary trauma, compassion satisfaction (ProQOL-5), generalized anxiety (GAD-7), and depression (PHQ-9): Mean

To identify the contribution of personaland work-contextual variables (gender work position, years of experience, and proximity to infected patients) in the dimensions of professional life $n=256$ Physicians: 85 satisfaction, burnout, and secondary trauma). To analyze the impact of thes dimensions on health workers' mental health.

$\begin{array}{ll} & \text { Nurses: } 133 \\ \text { Others: } 47 \\ \text { Men: } 84 \\ \\ \text { Women: } 181\end{array}$
DD $=7.0$. Burnout scores $(b=0.400, p<0.001)$ burnout score $M=19.8 \pm 5.0$. Mean secondary trauma score $M=18.0 \pm 5.6$. Average $S D=7.0$. Burnout scores $(b=0.400, p<0.001)$ and secondary traumatic stress $(b=0.200$ $p=0.007$ ) showed a significant positive association with depression scores. Frontline activity $(b=1.760, p=0.008)$ and work in an ICU $(b=2.290, p=0.001)$ was significantly associated with anxiety and depression scores. The mean anxiety and depression scores were $M=4.2$ and $M=4.5$. 
Table 1. Cont.

Author and Year Aims
Country

$n=240$

To determine the effect of the levels of stress, anxiety, and exhaustion on the quality of life of health professionals who actively worked in hospitals during the COVID-19 pandemic.
Sample

\section{Results}

Burnout (MBI) anxiety (STAI) quality of life (OLS) and stress (PSS): Considering their occupational situations, the mean burnout scores of nurses were significantly higher $(M=26.98 \pm 8.91, t=2.564$,

$p=0.004)$ than those of physicians $(M=29.34 \pm 8.41)$ and auxiliary personnel $(M=24.15 \pm 8.14)$. Burnout was significantly positively correlated with stress $(r=0.46, p<0.001)$ and trait anxiety $(r=0.59$, $p<0.001)$, and negatively with quality of life $(r=-0.700, p<0.001)$. Stress $(t=-2.392, p=0.017)$, trait anxiety $(t=3.214, p=0.001)$, and quality of life $(t=0.73, p=0.011)$ scores were higher in women than in men.

Burnout (CMBI), generalized anxiety (GAD-7), and depression (PHQ-9): Work burnout was a risk facto for anxiety (odds ratio OR $1,939(1,276-2,988)$ and depression (OR 3, $121(2,028-4,913)) ; 150(16.63 \%)$ depresion (OR 3,121 (2,028-4,913)); 150 (16.63\%) health workers experienced moderate-severe anxiety and $165(18.29 \%)$ had symptoms of moderate-severe depression; $36.2 \%$ simultaneously had anxiety and depression. The rates of occurrence of

depression symptoms in healthcare and to identify the associated risk Men: 283

Women: 619

$n=12,596$

ICU: 660

Pulmonary medicine: 419

To assess trauma, burnout, growth, and associated factors in nurses who worked Several countries

Pulmonary medicine: 419

Infectious diseases: 208

Emergencies: 702

Men: 555

Women: 12,041 moderate-severe anxiety and depression were significantly higher in those who experienced increased workloads $(p=0.001$ and $p<0.001$ respectively).

Burnout (MBI) and post-traumatic growth (PTGI-SF): Burnout: the depersonalization score was moderate $M=5.5 \pm 4.6$. Women, critical unit workers, and staff in departments related to COVID-19 had significantly higher depersonalization scores $(p<0.001)$. The average score for lack of personal accomplishment was $M=19 \pm 8.4$; participants experienced a small was $M=19 \pm 8.4$; participants experienced a smat
lack of personal accomplishment. Post-traumatic growth: the mean post-traumatic growth score was $M=28.0 \pm 11.5$; participants scoring in the 60th percentile or higher showed personal growth because of the effects of COVID-19. 
Table 1. Cont.

Author and Year Aims
Country

To assess the prevalence of burnout among physicians and nurses in the emergency services and urgent care centers of a regional health group through a cross-sectional study.
United States of America

$n=337$

Nurses: 210

Women: $67.7 \%$
Sample

Burnout (CBI): The mean personal exhaustion Burnout (CBI): The mean personal exha score wa $49.2 \pm$. N 6 , ranging from moder

$(M=51.3 \pm 19.6)$ than physicians

$(M=51.3 \pm 19.6)$ than physicians
$(M=45.7 \pm 16.2 ; p=0.005)$. Staff who had worked in emergencies or in an urgent care center prior to the COVID-19 pandemic also had a higher rate of moderate-severe personal burnout compared to those deployed from other departments $(90.4 \%$ vs. $9.6 \%, p=0.004)$.

Burnout (Mini-Z MBI), TEPT (IES-R), and generalized anxiety (GAD-7): Burnout was reported in $24(14.7 \%)$ participants, and in a significantly higher number of women compared to men ( $p=0.007) ; 4.9 \%$ obtained a score equivalent to probable PTSD; 74 participants $(45.5 \%)$ presented anxiety symptoms, with $25.8 \%$ in a mild range, $11.7 \%$ in a moderate range, and $8.0 \%$ in a severe range. Women reported a greater increase in anxiety symptoms compared to men $(p=0.001)$.

Burnout (Mini-Z MBI), generalized anxiety (GAD-7), and depression (PHQ-2): Burnout was reported in $76(21.8 \%)$ participants. The burnout prevalence was higher in residents compared to doctors (49 [29.7\%] vs. 27 [14.7\%], $p=0.001)$. Women had a higher prevalence compared to men (40 [29.2\%] vs. 36 [17.0\%], $p=0.010)$. Some 210 participants $(60.2 \%)$ had depression: $32.7 \%$ mild, $20.9 \%$ moderate, and $6.6 \%$ severe. Women had higher scores than men $(p=0.001)$. A total of 167 participants presented symptoms of anxiety $(47.9 \%)$ : $28.9 \%$ mild, $11.5 \%$ moderate, and $7.4 \%$ severe. Women had higher scores $(p=0.001)$, and 37 participants $(10.6 \%)$ met the criteria for a diagnosis of depression. 
Table 1. Cont.

Sample

$n=1160$

Gain insight into the experience of

Coleman et al. (2020) [52] arly-career surgeons and surgery

the American College of Surgeons.

Early-career surgeons: 695
Women: $47 \%$

To identify the main psychosocial variables

burnout in physicians and nurses in the first 2 Portugal

months of the COVID-19 pandemic in

Portugal. $n=497$
Physicians: 229 ( $48 \%$ men and 52\% women) Nurses: 268 (26.9\% men and $73.1 \%$ women $)$

\section{Results}

(PHO-9): $39 \%$ depersonalization, and $45 \%$ a decrease in the sense of personal accomplishment. Similarly, $56 \%$ of early-career surgeons reported emotional exhaustion $30 \%$ depersonalization, and $45 \%$ a decreased sense o personal accomplishment. Some $31 \%$ of the residents reported cepressed in $54 \%$ abits $22 \%$ ch apetite, $31 \%$ deceas interest or happiness in activities, $39 \%$ a change in weight, and $35 \%$ had difficulty maintaining attention. In turn, $31 \%$ of early-career surgeons reported symptoms of depression, $61 \%$ anxiety, $42 \%$ changes in sleep habits, $21 \%$ changes in appetite, $36 \%$ a lack of interest, $44 \%$ a change in weight, and $34 \%$ a decrease in the maintenance of care.

Burnout (OLBI), empathy (BES), and work area (AWS): Emotional exhaustion: physicians Depersonalization: physicians $(M=2.69+0.71)$ nurses $(M=2.76 \pm 0.70)$. Physicians: there were statistically significant negative correlations between burnout and depersonalization, and income; confidence in COVID-19 policies; peer justic istributive justice, procedural justice; and professional identification. There was a positive significant association between workload, and burnout and disengat meaningful work, patient fairness, and patient

disconnection. Male sex was also a risk factor for disconnection. Nurses: significant negative correlations for burnout and depersonalization, and income; confidence in COVID-19 policies; meaningful work; peer justice; patient justice; family patient justice; distributive justice; procedural justice and professional identification. Burnout was positively associated with affective empathy and negatively associated with age, years of professional experience, and COVID-19 task changes. Female sex was a risk factor for burnout. Disconnection was signifisnty natively related to religion. Burnout workload. 
Table 1. Cont.

Author and Year

Cravero et al. (2020) [54]

To determine how the level of exposure

to COVID-19 patients affected the

perceived safety, training, and

well-being of residents and fellows.
Country

$n=1420$

Medical residents: 1101

Physicians: 319

Medical residents exposed to $>60$

COVID-19 patients: 111

Physicians exposed to > 60 COVID-19

patients: 14

Men: $51 \%$

Women: $49 \%$
To assess the psychopathological impact of the pandemic on the general

population of Lombardy and compare

the prevalence of psychiatric symptoms

among the general population and

Italy

healthcare workers there. $n=432$

General population: 309

Health workers: 123

Men: $28 \%$

Women: $72 \%$

\section{Results}

Burnout (MBI): $66 \%$ of the residents who cared for more than 60 COVID-19 patients had

burnout, while $39 \%$ of the residents who did not see COVID-19 patients reported burnout $(p<0.001)$. The number of patients attended to was a predictor of burnout: attending to $>60$ COVID-19 patients (adjusted odds ratio

$[\mathrm{AOR}]=4.03 ; 95 \%$ confidence interval $[\mathrm{CI}$;

2.12-7.63]). Access to adequate PPE 'most of the time' $(\mathrm{AOR}=1.99 ; 95 \% \mathrm{CI}[1.41-2.80])$ or 'sometimes' (AOR $=281 ; 95 \%$ CI [1.60-4.91]) was significant

Burnout (MBI), anxiety and depression

(DASS-21), and insomnia (PQSI): $38 \%$ of health workers had symptoms of emotional

exhaustion, $39.8 \%$ depersonalization, and $48 \%$ exhaustion, 39.8\% depenson andization, and personal accomplishment. In the general population, $9.06 \%$ had symptoms of emotional exhaustion, $49 \%$ depersonalization, and $42.9 \%$ high personal accomplishment; A total of 59 health workers presented pathological levels of stress $(48 \%), 47$ anxiety $(38.2 \%), 51$ depression $(41.5 \%)$, and 88 'poor sleep' (71.5\%); 28

participants from the general population presented pathological levels of stress (57.1\%) 23 anxiety $(46.9 \%), 25$ depression $(51 \%)$, and 35 'poor sleep' (71.4\%).

Burnout (MBI): Levels did not change significantly in the period considered (emotional exhaustion $p=0.632$;

depersonalization $p=0.155$ ). Getting tested for COVID-19 and the number of shifts worked

was associated with high emotional exhaustion was associated with high emotional exhaustion and high depersonalization levels (OR 1.3; $95 \%$ respectively). Well-being was influenced by PPE, safety measures, the number of patients in their care, and training on the disease (data not specified). 
Table 1. Cont.

Author and Year Aims

Country

To explore the relationships between the dimensions of exhaustion and various psychological characteristics in Italian primary care physicians during the COVID-19 emergency.

\section{Italy}

$n=102$ primary care physicians

Men: 38

Women: 64
To measure the prevalence of burnout

syndrome during the COVID-19

pandemic in resident physicians.
Romania
Dimitriu et al. (2020) [58]
To investigate the effectiveness of implementation of the emotional

freedom technique (EFT) program in the Turkey

prevention of stress, anxiety, and

burnout in nurses treating COVID-19. $n=100$

Group A

Emergency residents: 30

ICU residents: 10

Radiology residents: 10

Group B

Gynecology residents:15

General surgery residents: 25
Burnout (MBI), coping with stressful situations (CISS), and resilience (RS-14): The coping style for stress emotions positively predicted emotional

exhaustion $(\beta=0.461, p<0.001)$. Task-oriented and emotion-oriented coping were significant predictors of depersonalization (respectively, $\beta=0.183$, $p=0.034 ; \beta=-0.298, p=0.023)$. Resilience positively predicted personal achievement $(\beta=0.500 ; p<0.001$ ). People with elevated levels of burnout showed less resilience and more often adopted a task-oriented coping strategy than the medium-risk group.

\section{Burnout (MBI)}

The overall prevalence of burnout syndrome among resident physicians was high. Burnout was significantly more common in residents who worked in regular services (group B) compared to resident physicians who worked in services considered frontline departments (group A; $86 \%$ vs. $66 \%$, $p=0.050$ ).

Burnout (Burnout Scale), Subjective relief services (SUD), and anxiousness (STAI): Burnout,

(SUD), and anxiousness (STAI): Burnout,
pre-intervention: EFT group $M=3.62 \pm 0.76$; contro $M=3.56 \pm 0.72$. Post-intervention: EFT group $M=2.48 \pm 1.06$; control $M=3.43 \pm 0.76$. Subjective relief services, pre-intervention: EFT group $M=7.82 \pm 1.33$; control: $M=7.48 \pm 1.36$. Post-intervention: EFT group $M=2.85 \pm 1.21$; control $M=7.40 \pm 1.53$. Anxiety, pre-intervention: EFT group $M=67.68 \pm 9.05 ;$ control $M=64.7 \pm=8.05$. group $M=67.68 \pm 9.05$; control $M=64.7 \pm=8.05$.
Post-intervention: EFT group $M=32.25 \pm 4.67$; control $M=64.43 \pm 7.68$. Significant differences were observed between the groups in the three variables studied after the intervention and between the pre-test and post-test in the EFT group $(p<0.001)$ 
Table 1. Cont.

Author and Year

Dinibutun (2020) [60]

To assess the prevalence and extent investigate factors related to burnou and the influence of COVID-19 on burnout syndrome.
Country

Sample

Results $n=200$ physicians

Men: $41 \%$

Women: $59 \%$
Burnout (MBI): emotional exhaustion: $M=3.00 \pm 0.62$, average levels; depersonalization: $M=2.00 \pm 1.01$ ), low levels; self-fulfillment: $M=2.00 \pm 0.57$ ), low levels; total burnout: $M=2.50 \pm 0.43$, low levels.

Burnout (PFI), TEPT (IES-R), depression (PHQ-9) resilience (CD-RISC10), and generalized anxiety (GAD-7): 83

participants (29.5\%) experienced burnout symptoms. The pates of burnout, depression, anxiety, and PTSD differed between professionals; senior medical staff reported the lowest levels of psychological distress. Frontline workers reported elevated levels of resilience and less severe psychological distress compared to other healthcare workers. Work in a high-exposure environment was associated with greater PTSD symptoms $(t(279)=226$ $p=0.024)$ and burnout $(t(270)=203, p=0.044)$. A subet $p=0.024)$ and burnout $(t(270)=2.03, p=0.044)$. A subset of participants experienced moderate-severe symptoms of depression (21\%), anxiety (20\%), and PTSD (29\%); 23 participants $(8.1 \%)$ felt suicidal ideation.

Burnout, secondary traumatic stress, and compassion satisfaction (ProQOL-5); depression and anxiety (DASS-21) and post-traumatic stress (PCL-C): Burnout levels were generally medium $(90.6 \%)$; the oldest participants in the sample (36-55 and <56) showed more burnout than the younger participants. Burnout was higher in physicians than in nurses and no difference was found between nurses and technicians/assistants. There were no differences in burnout between those who had or had not been in direct $n=973$ post-traumatic stress symptoms among flattening of the COVID-19 infection rate

Dosil et al. (2020) [62]

curve. To study the possible differences depression, compassion fatigue, and in these symptoms according to
relevant factors such as age, sex,

relevant factors such as age, sex,
professional category, contact with

compliance with health measures. in these symptoms according to other

COVID-19, and perception of social

\section{Spain}

Nurses: $318(32.6 \%)$

Technicians/assistants: $222(22.9 \%)$

Men:165 (16.5\%)

Women: $808(82.9 \%)$ contact with COVID-19. Secondary traumatic stress levels

were low $(0.2 \%$ high and $19.2 \%$ medium). Compassion satisfaction levels were high $(33.2 \%)$ or medium $(63.1 \%)$. The highest levels of compassion satisfaction were found among participants aged $26-35$ and $36-55$ years.

Compassion satisfaction was higher in

technicians/assistants than in nurses, while it was higher in nurses than in physicians. Levels of depression, anxiety, stress, and post-traumatic stress were significantly higher in nurses and technicians/assistants than in physicians. 
Table 1. Cont.

Author and Year Aims

Country

$n=2008$

Healthcare technicians: 707 (35.2\%)

Physicians: $511(25.4 \%)$

Nurses: $409(20.4 \%)$

assess the relationship between sociodemographic and mental health three dimensions of exhaustion:

Portugal

personal, work-related, and

client-related.
Pharmacists: $88(4.4 \%)$

Psychologists: $83(4.1 \%)$

Nutritionists: $72(3.6 \%)$

Men: $330(16.4 \%)$

Women: $1678(83.6 \%)$ $n=532$

Internal medicine: $223(41.9 \%)$

To determine the prevalence of burnou among hospital healthcare workers in

Elhadi et al. (2020) [64]

Libya during the COVID-19 pandemic

Libya

and the ongoing civil war.
Intensive care: $64(12 \%)$

Emergencies: $111(20.9 \%)$

Surgery: $134(25.2 \%)$

(a) $294(55.3 \%)$

Women: $238(44.7 \%)$

Burnout (CBI): 1066 participants (more than $50 \%$ ) showed high levels of work-related

burnout. Potential risk predictors for burnout

were gender, parental status, marital status,

and salary reduction. A higher level of

exhaustion was found in women compared to men $(p<0.001)$. Frontline workers showed higher levels of personal, work-related, and patient-related burnout ( $\beta=4.24, \beta=3.91$, and $\beta=2.35, p<0.001$, respectively). Workers in direct contact with COVID-19 patients presented higher levels of personal $(\beta=3.27$, $p<0.001)$ and work-related exhaustion $p<0.001)$ and work
$(\beta=3.45, p<0.001)$

Burnout (aMBI): Emotional exhaustion: 357 $(67.1 \%)$ participants reported high levels

$(67.1 \%)$ participants repoted high levels $\geq 10)$, the average score was $11.3 \pm 4.8$. A statistically significant association was found between emotional exhaustion and gender, years of work experience, service, and life in a conflict area $(p<0.050)$. Depersonalization: 25 $(47.4 \%)$ participants showed elevated levels $(\geq 10)$; the average score was $8.5 \pm 5.1$. The influencing characteristics were sex, age, department, internal displacement, and verbal abuse $(p<0.050)$. Personal accomplishment: $121(22.7 \%)$ participants showed a lower sense of personal achievement $(\leq 10)$; the average of personal achievement $(\leq 10)$; the average
score was $12.7 \pm 3.7$, but did not significantly correlate with other variables.

$n=84$

Nursing assistants: 33

To measure the level of burnout in health

El Haj et al. (2020) [65 workers in a geriatric nursing home

France
Nursing

Physicians: 15

Others: 1

Men: 25

Women: 59
Burnout (OLBI): Nursing assistants:

$M=37.66 \pm 3.32$; nurses: $M=38.89 \pm 3.32$;

physicians: $M=37.21 \pm 3.19$,

others: $M=39.42 \pm 3.76$

No significant differences were found between professional categories: $\chi^{2}(1, n=84)=0.36$ $p=0.550$ 
Table 1. Cont.

Author and Year

To investigate stress, burnout, and compassion fatigue in healthcare rorkers.
Italy

Country
Sample

\section{$n=102$}

Resident physicians: 12 (11.76\%)

Psychologists: 5 (4.90\%)

Nurses: $24(23.52 \%)$

Therapists (psychiatry, respiratory therapy, physical, occupational, and speech): $21(20.59 \%)$

Clinical social workers: 30 (29.41\%)

Technicians: 4 (3.92\%)

Administrative: $6(5.88 \%)$

Men: 48

Women: 54

$n=102$

Resident physicians: $12(11.76 \%)$

Psychologists: $5(4.90 \%)$

Nurses: 24 (23.52\%)

Therapists (psychiatry, respiratory

To investigate stress, burnout, and compassion fatigue in healthcare

Italy workers.

(20.59\%)

Clinical social workers: 30 (29.41\%)

Technicians: 4 (3.92\%) Administrative: 6 $(5.88 \%)$

Men: 48

Women: 54

$n=330$

Physicians: 140

To identify the prevalence of burnou and psychological suffering in health

Giusti et al. (2020) [68]
Italy professionals during the early phases of

the COVID-19 pandemic.
Nurses: 86

Nursing assistants: 38

Psychologists: 35

Others: 32

Men: 124

Women: 206

\section{Results}

Burnout (CBI), secondary trauma (STSS), and compassion fatigue (FCs): The overall

compassion fatigue scores were increased in all

the workers; the largest increase was in

psychiatric and multidisciplinary health

workers ( $22 \%$ and $33 \%$, respectively). The job

category with the highest percentage of

burnout $(39.67 \%$ vs. $40.67 \%)$ was social

workers in the psychiatric and

multidisciplinary departments. Compassion

fatigue was reduced among therapists and

nurses, and the scores were also lower on the

nurses, and the scores were also lower on the burnout and secondary trauma subscales
compared to physicians and psychologists.

Burnout (CBI), compassion fatigue (FCs), professional quality of life (ProQOL-5), and hopelessness (BHS): Nurses showed a high percentage of compassion fatigue and the

lowest average scores on the ProQOL subscale of vicarious traumatic stress $(45.83 \%$ with moderate to severe scores). There was a greate increase in the mean values of burnout in all the groups analyzed $(p=0.003)$. About half of the groups analyzed $(p=0.003)$. About half of
the respondents scored above average $(37.5 \%)$ on the hopelessness scale.

Burnout (MBI), anxiety and depression

(DASS-21), and TEPT (IES-6): Emotional

exhaustion: $M=22.3 \pm 11.4 ; 107(35.7 \%)$ had moderate scores, and $105(31.9 \%)$ had severe levels of emotional exhaustion. Anxiety: $M=3.3 \pm 3.6$. there were clinical levels of anxiety in $103(31.3 \%)$ participants. Depression $M=4.0 \pm 4.2$. clinical levels of depression were identified in 88 participants $(26.8 \%)$.

Post-traumatic stress: $M=3.2 \pm 2.1 ; 36.7 \%$ of the participants reported post-traumatic stress. 
Table 1. Cont.

Author and Year

Hoseinabadi et al. (2020) [69]

To assess the level of burnout during the

COVID-19 outbreak and identify the

influencing factors in frontline nurses

and non-frontline nurses.
Country

$n=245$ nurses

COVID-19 exposure:

Men: 82 (54.3\%)

Women: $69(45.7 \%)$

No COVID-19 exposure:

Men: 45 (52.1\%)

Women: $49(47.9 \%)$
To assess mental health (burnout, anxiety, depression, and fear) and its associated factors among frontline nurses caring for COVID-19 patients in Wuhan, China.
China

$n=2014$ nurses

Men: $260(12.9 \%)$

Women: $1754(87.1 \%)$
To assess the burnout and resilience of frontline emergency nurses in a tertiary

care facility.
India $n=120$ nurses

Men: $32(26.7 \%)$

Women: $88(73.3 \%)$

\section{Results}

Burnout (OLBI): The occupational stress

$(p=0.006)$ and burnout $(p=0.002)$ scores in the

exposure group were significantly higher than

in the non-exposure group. Work stress was

the only factor that was significantly linked to

COVID-19-related burnout ( $\beta=0.308$,

$p=0.031)$

Burnout (MBI), anxiety (SAS), depression

(SDS), self-efficacy (GSS), resilience

(CD-RISC-10), social support (MSPSS), and fear

(FS-HPs): Emotional exhaustion

$(M=23.44 \pm 13.80)$ correlated positively with

skin lesions $(r=0.182)$ and negatively with

self-efficacy $(r=0.193)$, resilience $(r=0.325)$

intra-family social support $(r=0.170)$, and

intra-family social support $(r=0.170)$, an

Depersonalization $(M=6.77 \pm 7.05)$ was

negatively correlated with resilience $(r=0.208)$

negatively correlated with resilience $(r=0.208)$,
intra-family social support $(r=0.221)$, and

extra-family social support $(r=0.216)$.

Personal accomplishment $(M=34.83 \pm 9.95)$

was positively correlated with self-efficacy

$(r=0.376)$, resilience $(r=0.436)$, intra-family

social support $(r=0.348)$, and extra-family

social support $(r=0.363)$; 67 obtained anxiety

scores $(3.3 \%)$, and 23 obtained severe

depression scores $(1.1 \%)$. Fear was negatively

correlated with resilience $(r=0.121)$.

Burnout (MBI-HSS) and resilience (CD-RISC):

Burnout: more than half of the nurses (54\%)

reported a high level of emotional exhaustion

and $37 \%$ reported a moderate level.

Approximately $52 \%$ of the participants

expressed a moderate level of

depersonalization; $78.5 \%$ experienced average

levels of personal accomplishment. Resilience:

$47.5 \%$ of the frontline nurses expressed a

moderate-high level of resilience, $53.3 \%$ a

moderate level of self-efficacy, and $45.8 \%$ a

moderate level of optimism. 
Table 1. Cont.

Author and Year Aims
Country

\section{Results}

Burnout (PFI), and depression and anxiety (DASS-21): The group of health workers exposed to COVID-19 had higher scores for burnout compared to those not exposed $(46.3 \%$ and $33.7 \%, p=0.011$ ). The exposed group had a higher prevalence of stress $(29.4 \%$ and $18.9 \%$, $p=0.016)$; there were no differences in anxiety $(21.6 \%$ and $14.9 \%, p=0.089)$ and both groups had similar rates of depression ( $28 \%$ and $26.3 \%$, $p=0.700$ ). Participants who were exposed to patients with COVID-19 reported significantly patients with COvID-19 reported significantly greater levels of stress compared to those who were not exposed to patients with COVID-19 $(10.96 ; 95 \% \mathrm{CI}[9.65-12.46]$ vs. $8.44 ; 95 \% \mathrm{CI}$ $[7.3-9.76] ; p=0.043)$.

\section{Burnout (PWLS), well-being (WBI), and} resilience (BRS): The following dimensions

To assess the well-being, resilience, burnout, and well-being factors of emergency physicians and residents during the phsich during the $n=213$

Emergency physicians: $157(74 \%)$

Residents: $56(26 \%)$

Men: $46 \%$

Women: $54 \%$ not significantly change (30\% to $22 \%$; $p=0.390)$; working part-time had twice the risk of burnout (OR 2.45; 95\% CI [1.10-5.47]); well-being improved ( $30 \%$ to $14 \% ; p=0.010)$; and symptoms of stress, anxiety, or fear were initially $83 \%$ and decreased to $66 \%(p=0.009)$. The initial resilience levels were normal-high.

Investigate the impact of the COVID-19 pandemic on the workflow, burnout, an career satisfaction of neurosurgery $n=111$ neurosurgery residents Burnout (aMBI): low levels of emotional exhaustion (51.4\%); low levels of residents in the US.

Men: 73

Women: 37 depersonalization (67.6\%); and elevated levels of personal achievement were noted $(78.4 \%)$.
Khalafallah et al. (2020a) [74] 
Table 1. Cont.

Author and Year

Khalafallah et al. (2020b) [75]

\section{To investigate the impact of the} pandemic on burnout and job

satisfaction among neurosurgeons in the

United States of America

Country

Sample

$n=407$ neurosurgeons

Neurosurgery subspecialties:

None: $150(36.9 \%)$

Spinal column/peripheral nerves: 87

$(21.4 \%)$

Cerebrovascular: 55 (13.5\%)

Pediatrics: 53 (13.0\%)

Neuro-Oncology: 37 (9.1\%)

Functional/stereotactic: $35(8.6 \%)$

Endovascular: $26(6.4 \%)$

Critical care: $15(3.7 \%)$

Men: $361(88.7 \%)$

Women: $46(11.3 \%)$

\section{$n=1117$}

Physicians: 1667

Nurses: 198

To study the prevalence of burnout due

India

to the COVID-19 pandemic in India.

Administration staff: 90

Paramedics (dietitians, physiotherapists,

pharmacists, etc.): 43

Others: 28

Men: $55 \%$

Women: $45 \%$
Burnout (aMBI): Most of the respondents reported low levels of emotional exhaustion

$(51.6 \%)$, low levels of depersonalization

$(87.5 \%)$, and high levels of personal

accomplishment (81.1\%). Neurosurgeons satisfied with their careers were less likely to have received subspecialty training in the spine/peripheral nerves $(p=0.028)$ and were ess likely to worsened because of COVID-19 $(p=0.045)$.

Burnout (CBI): The mean scores were as follows: personal exhaustion:

$M=49.72 \pm 18.68$; work exhaustion:

$M=39.69 \pm 20.43$; and exhaustion related to

the pandemic: $M=51.37 \pm 15.12$. The

prevalence of personal exhaustion ( $41.3 \%$ vs.

prevalence of personal exhaustion $(41.3 \%$ vs. $29.1 \%)$ was significantly higher $(p<0.010)$ among surveyed women; compared to men, the odds ratio of experiencing personal- and work-exhaustion were $1.35,95 \%$ CI [1.13-1.61] $(p<0.010)$, and $1.24,95 \% \mathrm{CI}[1.01-1.50]$ $(p<0.030)$, respectively.

Secondary trauma, fear, intention to change shift rotations, and leadership support

(ad-hoc): The results of the regression analysis showed that fear of COVID-19 had had a positive and significant effect on secondary trauma $(b=4.84, p<0.050)$, psychological distress $(b=4.83, p<0.050)$, and nurses
drauma distress $(b=4.83, p<0.050)$, and nurses'
intention to change their shift rotation $(b=4.79$ intention to change their shift rotation $(b=4.79$ $p<0.050)$. Furthermore, secondary trauma and the intention to change the shift rotation we
To examine the impact of the fear of

COVID-19 on nurses' intention to

trauma, and psychological distress. $n=380$ nurses

Men: 60

Women: 320 
Table 1. Cont.

Author and Year

Aims

Country

To assess the psychological impact of COVID-19 on French community

pharmacists.

\section{France}

$n=135$ pharmacists

Men: 57

Women: 78

\section{Results}

Burnout (MBI) and TEPT (IES-R): The mean scores were PTSD $=20.6 \pm 15.1$; emotional exhaustion $=23.0$

\pm 11.4 ; depersonalization $=10.9 \pm 5.5 ;$ and person \pm 11.4; depersonalization $=10.9 \pm 5.5$, and personal accomplishment $=48.1 \pm 7.2$. A total of 23 pharmacists (17\%) reported post-traumatic stress; 33 (25\%) emotional exhaustion; 46 (34.9\%) depersonalization; and $4(3 \%)$ low personal accomplishment. Women scored higher than men for post-traumatic stress disorder $(p<0.010)$ and depersonalization $(p<0.001)$.

Burnout (MBI) and anxiety towards death (patients' anxiety about death): $58.6 \%$ showed low levels of emotional exhaustion and $41.4 \%$ medium-high levels,

To find out if health professionals have suffered anxiety in relation to the death processes of their patients, and what variables were involved in this what $n=157$

Physicians: $22(14.0 \%)$

Nurses: $109(69.4 \%)$

Others: $26(16.6 \%)$

Men: $33(21.0 \%)$

Women: $124(79.0 \%)$

$31.8 \%$ showed low levels of depersonalization, while $68.2 \%$ presented medium-high levels; $45.9 \%$ showed low levels of personal accomplishment; $54.1 \%$ showed medium-high levels. The risk of suffering anxiety about the death processes of patients increased by 3 points in the presence of moderate-high levels of emotional exhaustion and depersonalization

Burnout (MBI-HSS): Both groups showed elevated levels of emotional exhaustion $(p>0.050)$. After 6 months, the indicators of emotional exhaustion $(p=0.019)$ and depersonalization $(p=0.028)$ in the IG were reduced compared to the CG; this was not the case for personal accomplishment $(p=0.067)$. After 12 months, the indicators of emotional exhaustion and depersonalization decreased and personal accomplishment increased in the IG, compared to the CG $(p<0.050)$.

To validate the effectiveness of strategy to reduce emotional

Kazakhstan exhaustion in a group of primary care physicians.

\section{$n=102$}

Intervention group (IG): 53

Control group (CG): 49

Men: 24

Women: 78 $n=740$

To study the level of vicarious trauma in the general population, frontline nurses, and nurses who were not on the frontline during the COVID-19 pandemic. (eneral population: 214

Nurses: 526

China

Frontline nurses: 234

Non-frontline nurses: 292

Men: 162

Women: 578 ficarious trauma (VTS): Vicarious trauma scores for and psychological responses, were significantly lower than non-frontline nurses $(p<0.001)$ and the general population $(p<0.001)$. No differences were general population $(p<0.001)$. No differences $w$
observed between the general population and non-frontline nurses $(p>0.050)$. 
Table 1. Cont.

Author and Year

Litam and Balkin (2020) [81]

o investigate how moral damage affected health workers during the

COVID-19 pandemic.

United States of America

Country

Sample

$n=119$

Physicians: 40

Nurses: 62

Other professions: 7

Men: 26

Women: 83

$n=830$

Physicians: 564

Nurses: 316

Men: 279

Women: 601
To assess the symptoms of

post-traumatic stress, anxiety,

depression, and levels of burnout an

resilience in Spanish health workers

during the COVID-19 pandemic, as well

as the relationship between these factors.
Spain

Men: 194

Women: 1228
Secondary traumatic stress (ProQOL-5) and moral damage (MIES): Secondary traumatic stress was significantly associated with moral damage,

representing only $8.4 \%$ of the variance in the model and with a negative relationship $(r=-0.49)$ with respect to moral damage. The increase in secondary traumatic stress was associated with a stronger probability of suffering moral injury.

Burnout (CMBI): A total of 80 (9.09\%) respondents showed emotional exhaustion, 445 (50.57\%) depersonalization, and $498(56.59 \%)$ had reduced personal accomplishment. There were no statistical differences between the three dimensions according to gender or occupational groups.

Burnout (MBI-HHS), TEPT (IES-R), anxiety and depression (HADS), and resilience (BRS): Emotional exhaustion: 584 (41\%) showed high scores; there were significant correlations $(p<0.050)$ with intrusion $(r=0.374)$, avoidance $(r=0.345)$, hyperarousal $(r=0.423)$, post-traumatic stress $(r=0.420)$, anxiety $(r=0.512)$, and depression $(r=0.484)$. Depersonalization: $216(15.2 \%)$ showed high scores; there were differences between men and women and significant correlations $(p<0.050)$ with intrusion $(r=0.171)$, avoidance $(r=0.219)$, hyperarousal $(r=0.218)$, post-traumatic stress $(r=0.225)$, anxiety $(r=0.289)$, and depression $(r=0.294)$. Personal accomplishment: $1164(81.9 \%)$ showed high scores; there were significant correlations $(p<0.050)$ with anxiety $(r=-0.160)$ and depression $(\mathrm{r}=-0.298)$. Anxiety: $295(20.7 \%)$ showed severe levels of disorder. Depression: 82 (5.3\%) showed severe disorder; post-traumatic stress: $805(56.6 \%)$ showed levels of psychiatric disorder. There were gender differences in the symptoms of PTSD, anxiety, and depression. The levels of resilience were moderate: $3.02 \pm 0.39$. 
Table 1. Cont.

Author and Year Aims
Country
To study whether the perception of threat generated by the COVID-19 pandemic explained burnout in nurses, and its moderating effect on the influence of resources and demands on

$\begin{array}{ll} & n=771 \text { nurses } \\ \text { Spain } & \text { Men: } 77 \\ & \text { Women: } 694\end{array}$

Women: 694 burnout.

Manzano-García and

\section{$n=157$}

To learn how the health crisis affected health professionals during the most critical weeks of the spread of the

Spain SARS-CoV-2 virus.

\section{Physicians:}

Nurses: $80(51.0 \%)$

Nursing assistants: 29 (18.5\%)

Others: $26(16.5 \%)$

Men: $33(21.0 \%)$

Women: $124(79.0 \%)$

\section{Results}

Burnout (CESQT) and psychosocial demand factors (UNIPSICO): Burnout:

factors (UNIPSICO): Burnout:
$M=42.39 \pm 11.39$. Autonomy $(r=-0.227$

$M=42.39 \pm 11.39$. Autonomy $(r=-0.22$

$p<0.010)$, social support $(r=-0.508$,
$p<0.010)$, and material and human resource

$p<0.010)$, and material and human resour
$(r=-0.404, p<0.010)$ were negatively

correlated with burnout; role conflicts

$(r=0.426, p<0.010)$, role ambiguity $(r=0.244$

$p<0.010)$, and work overload $(r=0.583$,

$p<0.010$ ), were positively correlated. The

perceived threat of COVID-19 was positively

correlated with exhaustion $(r=0.68 ; p<0.010)$

and was highest for burnout and the variables used to explain it.

Burnout (MBI): Emotional exhaustion: low,

$58.6 \%$; medium, $21.0 \%$; high, $20.4 \%$.

Depersonalization: low, 31.8\%; medium, 29.3\%

high, $38.9 \%$. Personal accomplishment: low,

high, $38.9 \%$. Personal accomplishment: low,
$45.9 \%$; medium, $34.4 \%$; high, $19.7 \%$. Need for

support: yes, $26.8 \%$; no, $73.2 \%$. Material

absence of protection increased stress/anxiety: yes, $85.4 \%$; no, $14.6 \%$.

Burnout (MBI): the overall prevalence of burnout was $31.4 \%$ (98 of 312 participants), and was higher in women (79 [80.6\%] vs. $144[67 \%]$ $p=0.020)$. The prevalence of burnout was significantly higher for nurses (OR 4.9; 95\% CI [2.2-11.2], $p=0.001$ ), laboratory personnel (OR $6.1 ; 95 \%$ CI [2.0-18.5], $p=0.002)$, radiologists (OR 16.4; $95 \% \mathrm{CI}[4.3-61.6], p=0.001$ ), and pharmacists (OR 4.9; 95\% CI [1.3-19.2] $p=0.020$ ). The prevalence of burnout $p=0.020$ ). The prevalence of burnout increased in those with less experience (OR $0.93 ; 95 \%$ CI [0.89-0.97], $p=0.001)$, and with greater anxiety due to a lack of familiarity with PPE (OR 2.8; 95\% CI [1.4-5.5], $p=0.002$ ). 
Table 1. Cont.

Author and Year

Miller et al. (2020) [86]

To determine the exhaustion and

resilience resources available in

respiratory care services.
Country

Burnout: In response to the single indicator "Have

you personally experienced burnout?", 72.4\%

reported having experienced burnout in the past

$32.6 \%$ had experienced burnout in the 6 months

prior; $32.5 \%$ did not use the resources available to

them for exhaustion; $11.3 \%$ took time off; $11.3 \%$

performed exercises; $8.1 \%$ meditated or practiced

mindfulness; $8.8 \%$ sought counseling/therapy or

used personal coping strategies; and $4.4 \%$ changed

their job.

Burnout (MBI), depression (BDI), and perceived stress (PSS): Mean scores for burnout: emotiona

exhaustion $=18.9 \pm 8.5$;

depersonalization $=7.3 \pm 4.5$; and personal

accomplishment $=11.4 \pm 5.0$. Mean score for

perceived stress $=31.4 \pm 8.7$, and for

depression $=16.0 \pm 9.4$. More stress was perceived

in public hospitals than in private ones

$(M=35.5 \pm 7.7, M=33.1 \pm 7.4, t=14.74, p<0.001)$

Nurses who were dissatisfied with the care they

had rendered had higher perceived stress

compared to those who felt competent

$(M=33.5 \pm 9.9, M=30.9 \pm 8.7, t=7.131, p=0.028)$

Nurses with a higher education degree who tested

positive for COVID-19 showed more symptoms of

depression $(p<0.050)$.

Burnout (MBI) and anxiety (GAD7): The

prevalence of burnout was $43.5 \%$, and of anxiety

$n=421$

$1.83 ; 95 \%$ CI [1.09-3.12], $p=0.024$ ), more anxious

(OR 5.92; 95\% CI [3.06-12.18], $p$ not specified), or

more fearful (OR 1.89; 95\% CI [1.23-2.93], $p=0.004$ )

were more likely to experience exhaustion. A

were more likely to experience exhaustion. A

perceived lack of support, public condemnation, a

and low confidence in health facility readiness

were associated with higher rates of burnout. 
Table 1. Cont.

Author and Year
Sample

$n=112$

General surgery: $48(42.8 \%)$

Neurosurgery: $18(16.0 \%)$

Orthopedic surgery: $12(10.7 \%)$

Plastic surgery: $10(8.9 \%)$

To measure the positive and negative

Osama et al. (2020) [89]

residency programs and on the lives of Pakistan surgical residents.
Cardiothoracic surgery: $4(3.6 \%)$

Other surgery: $6(5.4 \%)$

Urology: $14(12.5 \%)$

Men: 67 (59.8\%)

Women: $45(40.2 \%)$
To investigate psychological distress in infectious disease physicians during the COVID-19 disease outbreak in the Republic of Korea.
Republic of Korea

$=115$ physicians

Men: 48

Women: 67

\section{Results}

Burnout (MBI): Emotional exhaustion decreased after the pandemic (during the peak: $M=6.31 \pm 1.62$; after:

$M=3.77 \pm 1.08 ; p=0.008$ ). Depersonalization decreased

after the pandemic (during the peak: $M=3.10 \pm 1.06$; after: $M=1.00 \pm 0.80 ; p<0.001$ ). Personal accomplishment decreased after the pandemic (during the peak: $M=5.33 \pm 1.44$; after: $M=3.56 \pm 1.21 ; p=0.002)$. Of the total number of residents, $97(86.6 \%)$ stated that their surgical practice duration had been negatively affected by the pandemic. A total of $92(82.1 \%)$ had their clinical exposure affected; $69(61 \%)$ were worried about transmitting the disease to their relatives; and $43(38.4 \%)$ said they were afraid of dying because of their direct exposure to the virus. The average number of work hours per week for surgical residents decreased (before: $M=81.10 \pm 6.21$; after: $M=49.16 \pm 6.25 ; p<0.001$ ) because of the COVID-19 outbreak.

Burnout (MBI-HSS), depression, anxiety, and stress (DASS-21): $90.4 \%$ of the respondents met the diagnostic criteria for burnout; $20(17.4 \%)$ met the criteria for depression, $23(20.0 \%)$ for anxiety, and $5(4.3 \%)$ for stress. Women had higher scores for burnout, depression, and anxiety than men.

Compassion fatigue and satisfaction (ProQOL-5); depression and anxiety (GAD-7); insomnia (ISI); and distress (IES-R): Compassion fatigue: average level $n=8$ (14.3\%); mild level $n=27(21.4 \%)$; moderate level $n=27$ $(21.4 \%)$; severe level $n=54(42.9 \%)$. Women ( $p=0.048)$,

$n=126$

Physicians: $44(34.9 \%)$ To determine the prevalence of depression, anxiety, insomnia, distress,

Pinho et al. (2020) [91] and compassion fatigue, as well as factors related to the presence of Paraguay symptoms.
Nurses: $29(23.0 \%)$

Others: $53(42.1 \%)$

Men: $22(17.5 \%)$

Women: $104(82.5 \%)$ nurses $(p=0.004)$, and physicians $(p=0.022)$, all had greater levels of compassion fatigue. The symptoms of depression $(p=0.023)$, anxiety $(p=0.035)$, insomnia $(p=0.024)$, and distress $(p=0.001)$ were more severe in women. insomnia symptoms were presented in participants who had not attended cases of respiratory infections $(p=0.014)$ Anxiety was significantly higher in nurses compared to other health professionals $(p=0.038)$. Age and depression $(r S=0.253, p=0.004)$, anxiety $(r S=0.228, p=0.010)$, and distress $(r S=0.175, p=0.0497)$ were significantly negatively 
Table 1. Cont.

Author and Year Aims

To assess the mental health

Prasad et al. (2020) [92

outcomes of health workers workir

during the COVID-19 pandemic.

United States of America

Country

Sample

$n=347$

Nurses: 248

Administrative staff: 63

Nursing assistants: 36

Men: 32

Women: 315

$n=241$
To investigate the early impact of the

COVID-19 emergency and

quarantine on the well-being,

working conditions, and working

practices of mental health staff and

professionals in Lombardy and

compare the findings with the

available data on health workers

facing the COVID-19 outbreak.

Psychologists: $73(30.3 \%)$

Counselors: $68(28.2 \%)$

Physicin: $28(11.6 \%)$

Physicians: $28(11.6 \%)$

Social workers: $15(6.2 \%)$

Nurses: $27(11.2 \%)$

Support workers: 7 (2.9\%)

Managers/coordinators: 7 (2.9\%)

Others: $11(6.6 \%)$

Men: 56 (23.2)

Women: $185(76.8 \%)$

\section{$n=426$}

To assess the levels of anxiety and

exhaustion, changes in life, changes

at home, and measures to relieve the

stress of emergency physicians in

Level of $\mathrm{med}$

Faculty: 236

Fellow: 19

Resident: 168

Men: 235

COVID-19 pandemic.
United States of America

\section{Results}

Burnout (MBI), anxiety (GAD-7) and depression (PHQ-2): Burnout: $30.0 \%$ reported burnout. Anxiety: $23.1 \%$

experienced severe distress; $69.5 \%$ experienced some type of anxiety; $68.6 \%$ indicated that their anxiety symptoms had made their work or daily routine at least "somewhat difficult" to maintain. Depression: $22.8 \%$ had symptoms of depression.

Burnout (MBI), anxiety (GAD7), and depression (PHQ 9): Burnout: $M=16.7 \pm 11.5$. Outpatient services workers reported a decrease in workload $(68.2 \%)$, while for inpatient services workers, the workload had remained the same $(37.9 \%)$ or had increased (41.4\%). Anxiety: $M=5.1 \pm 3.4$; $11.6 \%$ had scores higher than moderate anxiety. Depression $M=4.7 \pm 2.9 ; 6.6 \%$ had scores higher than moderate depression. The factors associated with burnout, anxiety, and depression were being a doctor or a woman, working and depression werking in close contact with COVID-19-infected users, working in outpatient services, and the perception of a medium or high risk of contracting COVID-19 at work.

Burnout (ad-hoc): The emotional exhaustion of emergency physicians increased during the pandemic: median of 3 before the pandemic (IOR $=2-4)$ and 4 after the pandemic $(\mathrm{IQR}=3-6)$.

Burnout, compassion fatigue, and compassion satisfaction (ProQoL-5), and stress (PSS-14): Physicians had higher scores for compassion fatigue (21.6 vs. 19.4, $p=0.014)$ and burnout (26.2 vs. $24.3, p=0.005$ ), compared to nurses

$n=506$

To assess compassion fatigue, Ruiz-Fernández et al. (2020) [95] $\quad \begin{aligned} & \text { burnout, compassion satisfactio } \\ & \text { and perceived stress in health }\end{aligned}$

Spain professionals during the COVID-19 Nurses: $78.7 \%$ health crisis in Spain
Physicians: $21.3 \%$

Men: $23.3 \%$
Women: $76.7 \%$
Nurses had higher compassion satisfaction scores ( 39.9 vs. $37.1, p=0.001$ ) compared to physicians. The perceived stress scores were similar in both professions. Professionals working in specific COVID-19 services and in emergency departments had higher scores for compassion fatigue $(M=24.3 \pm 8.1)$ and burnout $(M=28.9 \pm 7.2)$. 
Table 1. Cont.

\section{Author and Year}

Sample

$n=583$

To know the prevalence of insomnia, fatigue, and psychological well-being (exhaustion, post-traumatic stress, and psychological distress), and examin the differences in these measures based on the working characteristics of nursing personnel during the COVID-19 pandemic in the USA.
United States of America Contact with COVID-19 patients:

No contact with COVID-19 patients:

Men: 25

Women: 396
To determine burnout levels and sleep quality in nurses during the COVID-19 Turkey pandemic. $n=267$ nurses

Men: 66

Women: 201

\section{Results}

Burnout (MBI), compassion fatigue (OFER-15), insomnia (PFI), post-traumatic stress (SPRINT), and psychological distress (PHQ-4): Burnout: emotional exhaustion: $M=32.21 \pm 12.01(n=451)$; depersonalization:

$M=11.13 \pm 6.99(n=452)$; personal accomplishment:

$M=32.95 \pm 8.00(n=450)$; nurses who cared for COVID-19 patients scored higher for depersonalization

$(t(400)=-2.750, p=0.006)$. Post-traumatic stress:

$M=15.32 \pm 7.00(n=502)$, indicating severe PTSD

symptoms; $55.38 \%(n=278)$ required an additional clinical evaluation; the nursing staff who cared for COVID-19 patients had higher levels of post-traumatic stress

$(t(402)=-3.276, p=0.001)$. Insomnia: $M=13.50 \pm 5.29$

$(t(402)=-3.276, p=0.001)$. Insomnia: $M=13.50 \pm 5.29$
$(n=564)$; the nursing staff who cared for COVID-19

$(n=564)$; the nursing staff who cared for COVID-19
patients had greater levels of insomnia $(t(388)=-2.064$

patients had greater levels of insomnia $(t(388)=-2.064$,
$p=0.040)$. Psychological distress: $M=6.10 \pm 3.30(n=422)$ $p=0.040)$. Psychological distress: $M=6.10 \pm 3.30(n=422)$,
indicating moderate levels of psychological distress; $47.39 \%$ $(n=200)$ of the sample presented possible depression, and $62.32 \%(n=263)$ possible anxiety.

Burnout (MBI) and sleep quality (PSQI): Emotional exhaustion $(p=0.040)$ and personal accomplishment $(p=0.019)$ were significantly higher in women. A negative relationship was observed between sleep quality and emotional exhaustion $(\mathrm{r}=-0.234, p<0.001)$ and depersonalization $(r=-0.174, p=0.004)$, but not with personal accomplishment $(p>0.050)$

Burnout (MBI), secondary traumatic stress (STSS), mental health (MHI-5), and insomnia (ISI): Insomnia was negatively related to exhaustion, $(r=0.390, p<0.001)$ and secondary traumatic stress ( $\beta=0.030 ; 95 \%$ CI $[0.000-0.084]$ ). Burnout was significantly positively related to mental health complaints $(r=0.560, p<0.001 ; \beta=0.310, p<0.001)$. health complaints $(r=0.560, p<0.001 ; \beta=0.310, p<0$
Secondary trauma was positively and significantly Secondary trauma was positively and significantly
correlated with insomnia $(r=0.59, p<0.001)$, exhaustion $(r=0.470, p<0.001)$, and mental health complaints $(r=0.38$ $p<0.001)$. The relationship between insomnia and mental health complaints was partially mediated by secondary traumatic stress and exhaustion. 
Table 1. Cont.

Author and Year

Soto-Rubio et al. (2020) [98]

Tan et al. (2020) [100]
Aims
To analyze the effect of psychosocial risks and emotional intelligence on burnout.
Country
Spain

Men: $20.9 \%$

Women: $79.1 \%$
Sample

Burnout (CESQT), emotional balance

(TMMS-24), and psychosocial risks

(UNIPSICO): The significant positive

predictors of burnout were emotional work

$(\beta=0.160, p<0.050)$, interpersonal conflict

$(\beta=0.170, p<0.050)$, role conflict $(\beta=0.440$,

$p<0.001)$, and emotional repair $(\beta=0.260$,

$p<0.001)$. High emotional attention increased

the predictive power of interpersonal conflicts

for burnout, while high emotional repair

reduced the predictive power of interpersonal

conflicts for burnout.

Burnout (MBI), generalized anxiety (GAD-7),

and depression (PHQ-9): Participants in the

flattened-infection-rate-curve group reported

mored-infection-ran

peak infections (332.5 and 294, respectively,

$p<0.001, r=-0.107)$. Anxiety among

participants from the peak infections group

was higher than for those after curve flattening

(831.5 and 370, respectively, $p<0.001, r=0.125$ ).

Both samples presented equal levels of the

symptoms of depression $(p=0.463$ ).

\section{Burnout (OLBI):}

$n=3075$

Physicians: 458 (14.9\%)

$\begin{array}{ll}\text { To assess burnout and its associated } & \text { Nurses: } 1394(45.3 \%) \\ \text { Singapore } & \text { Others: } 483(15.7 \%)\end{array}$

Men: $794(25.8 \%)$

Women: $2199(71.5 \%)$

Others: $82(2.7 \%)$
The mean exhaustion scores were $M=2.50$ and were higher for nurses $(M=2.52)$. The mean depersonalization scores were highest for administrative staff $(M=2.46)$ and lowest for support staff $(M=2.32)$ 
Table 1. Cont.

To analyze the psychological adjustment of Italian healthcare professionals durin the peak of the COVID-19 pandemic terms of perceived stress, anxiety, depression, and professional quality of life. $n=627$

Healthcare professionals exposed to

COVID-19 patients: 306

Healthcare professionals not exposed to

COVID-19 patients: 321 Burnout, compassion fatigue and vicarious (HADS); and perceived stress (PSS-10):

Healthcare professionals who did not work Healthcare professionals who did not work
with COVID-19 patients had lower burnout scores $(M=26.38 \pm 6.76)$ compared to health workers who worked with COVID-19 patients $(M=29.70 \pm 7.35 ; t=24.01, p=0.037)$.

Healthcare professionals exposed to COVID-19 patients showed significantly higher levels of stress $(t=8.47, p=0.013)$, burnout $(t=24.01$, $p=0.037)$, secondary trauma $(t=18.74$ $p=0.002)$, anxiety $(t=8.59, p=0.014)$, and $p=0.002)$, anxiety $(t=8.59, p=0.014)$, and
depression $(t=8.51, p=0.013)$. No differences depression $(t=8.51, p=0.013)$. No differente
in compassion satisfaction were detected. Those working in the Italian regions most affected by the pandemic experienced higher levels of perceived stress $(t=7.93, p=0.013)$ and burnout $(t=5.30, p=0.008)$, and lower levels of compassion satisfaction $(t=5.28$, $p=0.008$ ).

Vicarious trauma (STSS-I) and coping with stress (CSES-SF): In the healthcare group, there were significant differences in the effect of stress in decision making $(F=3.680 ; p<0.050)$, which was higher in physicians

$n=210$

To identify the coping strategies used by emergency and healthcare professional to deal with stressors related to the COVID-19 pandemic that may be associated with the risk of developing vicarious trauma.
Healthcare group:

Physicians: 57 (50\%)

Nurses: 47 (37.3\%)

Psychologists: $9(7.14 \%)$

Nursing assistants: 7 (5.56\%)

Emergency group:

Firefighters: $21(23.6 \%)$

Civil protection: $20(22.5 \%)$

Men: $90(42.9 \%)$

Women: $120(57.1 \%)$
$(M=14.51 \pm 2.89)$ than psychologists

$(M=11.11 \pm 2.15 ; p<0.050)$, as well as for $(M=11.11 \pm 2.15 ; p<0.050)$, as well as for stress due to COVID-19 $(F=3.57, p<0.05)$, which was higher in nurses $(M=16.19 \pm 3.47)$ than doctors $(M=14.30 \pm 3.61 ; p<0.050)$. There were no differences in the levels of stress and secondary trauma or coping strategies in the emergency group. Compared to men women reported greater levels of physical stress $(M=10.90+4.83$ vs. $M=7.30 \pm 4.57$ $t=5.470, p<0.001)$, higher $M=7.30 \pm 4.57$, $t=5.47, p<0.001)$, higher emotional stres $(M=13.30 \pm 3.68$ vs. $M=11.64 \pm 3.80$, $t=3.180, p<0.010)$, and higher stress caused by COVID-19 ( $M=14.93 \pm 3.68$ vs. $M=13.58 \pm 4.22, t=2.480, p<0.050$. 
Table 1. Cont.

\section{Author and Year}

Country

Physicians: $41 \%$

Nurses: $40 \%$

Others: $19 \%$

Men: $35 \%$

Women: $65 \%$

\section{Results}

Burnout and emotional distress (ad-hoc): Emotional distress and burnout were significantly associated with female sex (absolute risk reduction [ARR] $=1.16$; $95 \%$ CI [1.01-1.33]) and being a nurse (ARR $=1.31 ; 95 \%$ CI 1.13-1.53]). Those who had seen $10-50$ or $>50$

COVID-19 patients had a $17 \%$ and $28 \%$ higher risk of exhaustion than those who had seen $<10$ patients. The limited availability of PPE and shortage of nurses were associated with a risk of burnout $>30 \%$ and $18 \%$, respectively. The most common concerns included transmitting the infection to family members $(61 \%)$, emotional distress and exhaustion (52\%), concerns about their own health $(44 \%)$, and experiencing social stigma from their communities (21\%). All health concerns were highest in North America. $n=190$

Frontline services:

Men: $28 \%$

Women: $72 \%$

Non-frontline services:

Men: $6 \%$

Women: $94 \%$
Burnout (MBI): the prevalence of exhaustion was significantly lower in the frontline group than in the non-frontline group $(13 \%$ vs. $39 \%$; $p<0.001)$. The prevalence of a low level of personal accomplishment was lower in the frontline group than in the non-frontline group (39\% vs. $61 \%$; $p=0.002$ ).

Burnout (MBI), perceived stress (PSS), depression (BDI) and resilience (RSA): there was a moderate positive relationship between depression and emotional exhaustion $(r=0.490, p<0.001)$. The risk of depression was 1.92 times higher in midwives than in nurses $(95 \%$ CI [1.08-3.41]. The mean scores for emotional To investigate the relationship resilience, burnout syndrome, an resilience, burnout syndrome,
sociodemographic factors and Turkey $n=377$ depression in midwives and nurses during the COVID-19 outbreak. exhaustion and depersonalization were significantly higher in midwives and nurses with high depression scores, but the personal achievement score was significantly lower in this group. The perceived stress score was significantly higher in midwives and nurses with high depression scores $(p<0.001)$. High

psychological resilience was protective against the risk of depression (OR $=0.95 ; 95 \%$ CI [0.93-0.96], $p<0.001$ ) 
Table 1. Cont.

Author and Year

Zerbini et al. (2020) [106]

\section{Aims}

Country

To explore whether people working in specific COVID-19 services were experiencing greater psychosocial stress compared to their colleagues working in regular services, and whether different healthcare professionals (nurses vs. physicians)

COVID-19 pandemic. were affected differently by the

Germany $n=111$

Sample

\section{Results}

Nurses: 75

Nurses working in specific COVID-19 services: 45

Nurses working in regular services: 30

Physicians: 35

Physicians working in specific

COVID-19 services: 17

Physicians working in regular services:

Burnout (MBI), depression, anxiety, and stress (PHQ): Compared to collos Compared cos wor higher levels of burnout $(t(73)=-2.970, p=0.004)$ and depressed $\operatorname{mood}(t(73)=-3.066, p=0.003)$; in addition their levels of personal accomplishment were lowe $(t(73)=3.246, p=0.001)$ compared to physicians.

Burnout was related to depression $(r=0.550, p<0.001)$ anxiety $(r=0.540, p<0.001)$, and stress $(r=0.540$,

$p<0.001$ ). Comparison between groups did not reveal statistically significant effects.

Burnout (MBI) and stress factors (ad- hoc): Burnout: emotional exhaustion: $M=12.27 \pm 7.14$;

depersonalization: $M=2.07 \pm 2.78$; personal

accomplishment: $M=16.44 \pm 8.36$. Lower age, less work experient and a longer time spent working work experience, and a longer time spent working in quarantine areas were all associated with higher levels of emotional exhaustion (statistics not specified). The subgroup that had spent the longest time working in quarantined areas had the highest levels of depersonalization (statistics not specified). Stress

To identify stressors and burnout in frontline nurses caring for

Zhang et al. (2020) [107]
COVID-19 patients in Wuhan and

Shanghai, and explore the perceived

and effective moral support

China

strategies. $n=107$ nurses

Men: 10

Women: 97 VID-19 questionnaire: homesickness $(96.3 \%)$, uncertainty about how long the current work status would last $(85.0 \%)$, concern about infecting oneself $(84.1 \%)$, skin damage caused by prolonged use MPE (75.7\%), and disc Main coping strategies: adopting preventive measures; learning about COVID-19, active learning of professional knowledge; adjusting attitudes and positively facing the COVID-19 epidemic; and talking with family and friends. Main effective supports: support from supervisors; sufficient supplies of material; subsidies provided by the government; clear instructions on treatment procedures; and adequate knowledge of COVID-19. 
Table 1. Cont.

Author and Year
To assess the psychometric properties of the 10-item Moral Injury Symptom Scale for health professionals (MISS-HP).
Country

Sample

$n=3006$

Nurses: 583

Physicians: 2423

\section{Results}

Burnout (MBI-HSMP), moral injury (MISS-HP) depression (PHQ-9), and generalized anxiety (GAD-7): Emotional exhaustion was correlated with moral damage $(r=0.340, p<0.050)$, depression $(r=0.620$, $p<0.050)$, anxiety $(r=0.620, p<0.050)$, and well-being $(r=-0.530, p<0.050)$. Depersonalization was correlated with moral damage $(r=0.400, p<0.050)$, depression $(r=0.590, p<0.050)$, anxiety $(r=0.570$, $p<0.050)$, and well-being $(r=-0.520, p<0.050)$.

Notes: aMBI = Abbreviated Maslach Burnout Inventory (Maslach et al., 1986); AWS = The Areas of Worklife Scale (Leiter and Maslach, 2004); BDI = Beck Depression Inventory (Beck et al., 1996); BES = Basic Empathy Scale (Jolliffe and Farrington, 2006); BHS = Beck Hopelessness Scale (Beck and Steer, 1993); BRS = Brief Resilience Scale--burnout (Smith et al., 2008); CBI = Copenhagen Burnout Inventory (Kristensen et al., 2005); CD-RISC = Connor-Davidson Resilience Scale 25 (Connor and Davison, 2003); CD-RISC-10 = Connor-Davidson Resilience Scale 10 (Campbell-Stills and Stein, 2010); CESQT = Spanish Burnout Inventory (Gil-Monte et al., 2019); CIS = Coping Inventory for Stressful Situations (Sirigatti, and Stefanile, 2009); CMBI = Chinese version of the Maslach Burnout Inventory (Zhou et al., 2016); CSES-SF = The Coping Self-Efficacy Scale-Short Form (Chesney et al., 2006); DASS-21 = Depression Anxiety, and Stress Scale 21 (Henry and Crawford, 2005). FCs = short Fatigue Compassion Scale (Adams, 2004); FS-HPs = Fatigue and Fear Scale for Healthcare Professionals (Sakib et al., 2020); GAD-7 = Generalized Anxiety Disorder Assessment-7 (Spitzer, 2006); GSS = Chinese version of the General Self-efficacy Scale (Zhang et al., 1995); HADS = Hospital Anxiety and Depression Scale (Zigmond and Snaith, 1983): IES-6 = Impact of Event Scale-Revised (Weiss, 2007); IES-R = Impact of Event Scale-Revised (Weiss, 1997); ISI = Insomnia Anxiety and Depression Scale (Zigmond and Snaith, 1983); IES-6 = Impact of Event Scale-Revised (Weiss, 2007); IES-R = Impact of Event Scale-Revised (Weiss, 1997$)$; ISI = Insomnia Jackson, 1986); MHI-5 = Mental Health Continuum—Short Form (Franken, et al., 2018); MIES = Moral Injury Events Scale (Nash et al., 2013); Mini-Z MBI = Single-Item Maslach Burnout Inventory (Linzer et al., 2016); MISS-HP = Moral Injury Symptoms Scale-Health Professional (Mantri et al., 2020); MSPSS = Multidimensional Scale of Perceived Social Support (Zimet et al., 1988); OFER-15 = Occupational Fatigue Exhaustion Recovery 15 (Winwood, 2006); OLBI = Oldenburg Burnout Inventory (Demerouti et al.,2003); PCL-C = Post-Traumatic Stress Scale (Weathers et al., 1991); PFI = Stanford Professional Fulfillment Index (Trockel, 2018); PHQ 9 = The Patient Health Questionnaire 9 (Kroenke et al., 2001); PHQ = Patient Health Questionnaire (Spitzer et al., 1999); PHQ-2 = The Patient Health Questionnaire 2 (Kroenke et al., 2003); PHQ-4 = The Patient Health Questionnaire 4 (Kroenke et al., 2009); PSQI = Pittsburgh Sleep-Quality Index (Buysse et al., 1989); ProQOL-5 = Professional Quality Of Life Scale version 5 (Stamm et al., 2009); PSS = Perceived Stress Scale (Cohen et al., 1983), PSS-10 = Perceived Stress Scale 10 (Cohen et al, 1983); PSS-14 = Perceived Stress Scale 14 (Cohen et al, 1983); PTGI-SF = Posttraumatic Growth Inventory-Short Form (Tedeschi, and RS-14 = 14-Item Resilience Scale (Wagnild, 2009); SAS = Chinese version of Zung's Self-Rating Anxiety Scale (Zung 1971); SDS = Chinese version of Zung's Self-Rating Depression Scale (Zung, 1965); SPRINT = Short Post-Traumatic Stress Disorder Rating Interview (Connor, and Davidson, 2001); STAI = Trait State Anxiety Inventory (Spielberger, 1970); STSQ = Secondary Traumatic Stress Questionnaire (Meda et al., 2012); STSS = Secondary Traumatic Stress Scale (Ting, 2005); STSS-I = Secondary Traumatic Stress Scale Italian Version (Setti and Argentero, 2012); SUD = Scale of Subjective Relief Units (Wolpe, 1990); TMMS-24 = The Trait Meta-Mood Scale (Salovey, 1995); UNIPSICO = Psychosocial Resource Factors (Gil-Monte, 2016); VTS = Vicarious Trauma Survey (Vrklevski and Franklin, 2008); WBI = Well-Being Index (Dyrbye, 2019). 
The research included in this review was all carried out between February and May 2020, with most of the studies having collected data between March and April (that is, Kannampallil et al. [72]; Ruiz-Fernández et al. [95]; Trumello et al. [101]). Most of the study samples included 100 to 400 participants; Chen et al. [48] included the largest number of participants (12,596 people), while the smallest study cohort was limited to 80 participants [59]. As shown in Table 1, physicians and nurses were the most-studied groups, either separately or together, during the health crisis caused by SARS-CoV-2. In addition, several researchers also focused on other medical professionals including residents, assistants, administrative personnel, physiotherapists, and laboratory technicians, among others. In terms of gender, the samples in $83.4 \%$ of the studies comprised more than $50 \%$ women, with only $16.4 \%$ of the articles including more men than women [39,41,42,50-52,54,56,64,74,89,94].

The impact of the COVID-19 pandemic on health professionals of different nationalities was also studied. The most-examined country was the United States of America, with 15 studies [38,49,50,52,56,65,72,73,75,81,86,92,94,104,109]. The two European countries in which the effects of COVID-19 were most studied were Italy and Spain, with eleven $[8,43,45,55,57,66-68,93,101,102]$ and seven articles $[44,62,78,82-84,95,98]$, respectively

Regarding the study variables, burnout was studied in $67(88 \%)$ of the papers included in this review, most often with the Maslach Burnout Inventory $[8,40,42,43,46,48,54-58,60,68,70,78,84,85,87-89,92,93,96,97,104-107,109,110]$. A total of $61 \%$ of the studies used the aforementioned questionnaire or one of its derivatives: the aMBI [52,74,75], CMBI [7,48], Mini-z MBI [50,51], MBI-HHS [39,71,82,90,108], or PWLS [73]. Other authors developed an ad-hoc questionnaire [94,103] or used instruments such as the CBI $[49,63,66,67,76]$, OLBI $[53,65,69,100]$, ProQOL $[45,62]$, PFI $[61,72,109]$ or CESQT [83,98]. The highest burnout found in the reviewed studies was been for infectious disease physicians in the Republic of Korea, with $90 \%$ of them presenting burnout [90]. The lowest burnout was found in a study carried out in Spain, in which burnout was present in $20.4 \%$ of health professionals [84].

The average level of burnout among healthcare professionals was high, especially on the emotional exhaustion and depersonalization subscales $[7,39,43,52,63,64,71,88]$. Some studies indicated high scores as a consequence of the pandemic on the personal accomplishment subscale [75,78], although these were lower in other studies $[7,39,52,60,84]$. Numerous reports pointed out the influence that some variables had on the perception of burnout, although 31\% (24) of the studies that evaluated burnout did not study its relationship with other variables. The most-studied variables were gender, profession, and workplace (COVID-19/frontline rooms vs. non-COVID-19/secondline rooms). Women showed higher scores on the emotional exhaustion and depersonalization subscales $[43,51,76,85,90,93,96,103,110]$.

Regarding the professional category, higher burnout scores were reported for nurses in several articles $[46,49,65,85,100,103]$, although others pointed towards higher levels of burnout among physicians [62,94]. In terms of the workplace, the results were also contradictory; some research indicated that health workers on the frontline against COVID-19 suffered less burnout $[58,104]$, while the majority found higher burnout scores among these same health workers $[40,63,69,72,93,95,101,106,109]$. Compared to the general population, healthcare personnel showed higher burnout scores $[55,80]$. The number of patients attended to also appeared to influence the level of exhaustion: the more COVID-19 patients seen by the participants, the higher their levels of exhaustion [54].

The risk factors, or those that showed a positive relationship with burnout, were anxiety and depression [82,106,108], insomnia [96,106], and moral damage [106]. Work stress also influenced burnout [69] and the lack of personal protective equipment affected emotional exhaustion $[39,57]$. Protective factors, or those whose presence was related to lower levels of burnout, included resilience and social support [70], and quality of life [46]. In addition, two studies observed that different interventions positively affected the levels of burnout in health workers. For example, Dincer and Inangil [59] implemented a program of emotional freedom techniques that reduced the level of burnout in healthcare personnel. 
Likewise, Lee et al. [79] found that following a coping strategies program resulted in lower levels of burnout among healthcare professionals. Finally, positive correlations were observed between burnout and secondary trauma or compassion fatigue [45].

Compassion fatigue was also studied in $16(21 \%)$ of the studies included in the review. Of note, some studies referred to the concept as compassion fatigue $[62,66,91,95,101,109]$ while others referred to it as secondary or vicarious trauma $[17,38,44,45,67,77,80,97,101,102]$. The instruments most used to assess these variables were the ProQOL-5 [45,62,66,81,91,95,101] and STSS $[17,97,102]$. The levels of compassion fatigue or vicarious trauma found in healthcare professionals were generally high $[17,44,45,91,101]$, although in specific studies they were medium [38] or low [62].

Regarding the protective and risk factors for compassion fatigue, again, the studies we considered focused on variables such as gender, profession, or workplace. Specifically, working with COVID-19 patients tended to increase secondary trauma scores $[44,109]$. Being a woman was also associated with higher levels of compassion fatigue [17,91]. Additionally, the professional category seemed to influence the perception of fatigue, although the results were inconclusive. Physicians showed higher compassion fatigue scores in the study by Ruiz-Fernández et al. [95]. Franza et al. [67] found that mental health workers had higher compassion fatigue scores, while the groups of therapists and nurses showed reduced compassion fatigue and lower scores on the burnout and secondary trauma subscales with respect to groups of physicians and psychologists. In any case, studies of this nature were scarce.

Finally, compassion satisfaction was only studied in four (5\%) of the studies included in this current review $[45,62,91,95]$ and the ProQOOL-5 questionnaire [23] was used to assess this factor in all these studies. In terms of the levels of compassion satisfaction, the study by Buselli et al. [45] found mean levels of $38.2 \pm 7.0$ for the sample of physicians and nurses. Along the same lines, Dosil et al. [62], reported high (33.2\%) or medium (63.1\%) levels of compassion satisfaction in health professionals. The latter authors also observed a relationship between compassion satisfaction and professional category, with higher levels of compassion satisfaction being reported in medical assistants/technicians compared to nurses and physicians. In contrast, Ruiz-Fernandez et al. [95] found that nurses had higher scores for compassion satisfaction than physicians. The last study that evaluated compassion satisfaction did not provide descriptive or inferential data in this regard [91].

\section{Discussion}

The main objective of this work was to understand the impact of the COVID-19 pandemic on the quality of life of healthcare professionals, specifically in terms of burnout, compassion fatigue, and compassion satisfaction. To this end, we carried out a systematic review of the literature produced during the first year of the pandemic (2020) which, after screening 2856 records, finally included 76 research papers. The main characteristics of the samples included in the reviewed studies agreed with those previously reported for health professionals in other systematic reviews, in which nurses and women predominated as the main participants [111-113]. Considering the results we obtained, it is evident that burnout is still used as the main indicator of emotional well-being in health professionals, much more so than other variables such as fatigue and compassion satisfaction that are used in the more recent literature. The data in this review coincided with those from Mol et al. [113], in which $88 \%$ of the articles they examined evaluated exhaustion, while compassion fatigue and compassion satisfaction was considered only in $21 \%$ and $5 \%$ of the cases, respectively.

Regarding the effect of the pandemic on health workers, we observed a worsening of the level of burnout. Specifically, in many of the studies $[7,39,43,52,63,64,71,88]$, the scores for emotional exhaustion and depersonalization exceeded the medium-high levels obtained in pre-pandemic reviews $[111,114,115]$. However, for certain professional profiles such as healthcare professionals in the oncology area, elevated levels of emotional exhaustion and depersonalization had already been identified prior to the pandemic [116]. Of note in this present review was the fact that, compared to previous reviews which described 
very heterogeneous prevalences ranging from $0 \%$ to $80 \%$ [117], the levels of burnout in the studies we considered were more homogeneous, ranging from $30 \%$ to $60 \%$ [39,43,55,71].

In the same way that burnout increased in health professionals during the COVID19 pandemic, an increase in compassion fatigue or vicarious trauma was also observed during the same period. Coinciding with the review by Xie et al. [118] implemented in emergency nurses before the COVID-19 pandemic, our results suggest that healthcare professionals had high scores for compassion fatigue [17,44,45,91,95,101], although other studies [38] have reported medium levels for this factor. In contrast, reviews conducted prior to the COVID-19 pandemic found moderate levels of compassion fatigue among healthcare workers [114,115]. However, very little data regarding compassion satisfaction were available, and some of these studies were inconclusive [91]. The levels of compassion satisfaction were generally medium or high [62], and were similar to those from before the COVID-19 pandemic. For example, the data collected by Xie et al. [118] from 2015 to 2019, found medium compassion satisfaction levels among oncology nurses.

Regarding the risk factors for developing burnout, our results indicate that the variables that influenced professional quality of life were gender (female sex), profession (nursing), and the workplace (attending or not attending patients with COVID-19). Indeed, the first two risk factors have already been recorded elsewhere in the literature $[111,119]$. Other variables that emerged as risk factors in this current review included anxiety, depression, and insomnia. Along the same lines, Gómez-Urquiza et al. [116] also highlighted these same risk factors for burnout. Furthermore, the same risk factors have also been observed for compassion fatigue. Finally, given the scarcity of results, we were unable to detect risk or protective factors for compassion satisfaction. In agreement with results from before the pandemic, the protective factors against burnout detected in this review included resilience, social support, and participating in interventions to reduce burnout. For example, in research on resilience and burnout, Heath et al. [120] found that preventive strategies, self-care, organizational justice, and having individual and organizational preventive strategies were protective factors against the development of emotional exhaustion. In fact, these interventions were already being implemented, and were equally effective before the COVID-19 pandemic [113].

Finally, it is worth highlighting both the strengths and limitations of this present study. Of note, although some literature reviews from prior to the pandemic focused on burnout among healthcare professionals, very few studies evaluated compassion fatigue, and even fewer studied the effect of compassion satisfaction. Reviews that focused on the impact of COVID-19 were much scarcer, with only one considering compassion fatigue and none having reviewed the literature on compassion satisfaction. Additionally, even though research focusing on burnout was more abundant, a much higher proportion of the relevant academic literature was considered in this present review. For example, the review by Sharifi et al. [35] on burnout among healthcare workers during the COVID-19 pandemic only included 12 studies; Chew et al. considered 23 studies evaluating emotional exhaustion and other variables; and Amanullah and Ramesh [32] only included five articles. Similarly, the only review available on vicarious trauma only included seven studies [36] compared to the 16 considered in this present review. Regarding the limitations of our work, we did not assess the quality of the articles we included in this review. Furthermore, to facilitate the synthesis of the results, we only included quantitative studies; therefore we may have excluded qualitative studies containing relevant information. In this sense, future work could assess the information collected in these qualitative studies.

\section{Conclusions}

Based on the results of our work, and in light of the literature we reviewed, we concluded that the quality of life of health professionals was significantly affected by the COVID-19 pandemic. Specifically, burnout levels increased from medium-high to high and compassion fatigue went from medium to high. Healthcare professionals reported high rates of emotional exhaustion, depersonalization, low personal accomplishment, 
and compassion fatigue, and low rates of compassion satisfaction. In addition, given that research from all five continents was included in this review, these findings can be considered global.

Therefore, in light of the results, we can say that the vulnerability of healthcare professionals to processes such as burnout or compassion fatigue has increased even more as a result of the COVID-19 pandemic. These problems, in addition to affecting the quality of care provided by health personnel, have a negative impact on professionals' well-being and quality of life; this can aggravate the lack of health professionals that health systems have suffered around the world for decades. Of note, the risk factors and protective factors have not changed compared to previous findings, meaning that we already have the scientific knowledge required to implement interventions to mitigate the empathic burnout of our healthcare professionals. We can no longer afford not to implement preventive strategies to prevent processes such as burnout and compassion fatigue in health professionals.

Supplementary Materials: The following supporting information can be downloaded at: https: / / www.mdpi.com/article/10.3390/healthcare10020364/s1, Supplement S1 PRISMA checklist, Supplement S2 Keywords and search terms used in the systematic review.

Author Contributions: Conceptualization, C.L. and L.G.; methodology, L.G. and C.L.; formal analysis, C.L., P.D. and L.G.; writing—original draft preparation, C.L. and P.D.; writing-review and editing, L.G. and N.S.; supervision, L.G.; project administration, L.G. and N.S.; funding acquisition, L.G. and N.S. All authors have read and agreed to the published version of the manuscript.

Funding: This research was funded by project RTI2018-094089-I00, ‘Longitudinal study of compassion and other professional quality of life determinants: national level research on palliative care professionals' (CompPal) [Estudio longitudinal de la compasión y otros determinantes de la calidad de vida profesional: Una investigación en profesionales de cuidados paliativos a nivel nacional (Comp Pal)] from the Ministerio de Ciencia e Innovación/Agencia Estatal de Investigación/FEDER.

Institutional Review Board Statement: Not applicable.

Informed Consent Statement: Not applicable.

Data Availability Statement: The data presented in this study are available within the article.

Conflicts of Interest: The authors declare no conflict of interest.

\section{References}

1. Pfefferbaum, B.; North, C.S. Mental Health and the COVID-19 Pandemic. N. Engl. J. Med. 2020, 383, 510-512. [CrossRef] [PubMed]

2. Liu, X.; Chen, J.; Wang, D.; Li, X.; Wang, E.; Jin, Y.; Ma, Y.; Yu, C.; Luo, C.; Zhang, L.; et al. COVID-19 Outbreak Can Change the Job Burnout in Health Care Professionals. Front. Psychiatry 2020, 11, 1362. [CrossRef] [PubMed]

3. Rajkumar, R.P. COVID-19 and Mental Health: A Review of the Existing Literature. Asian J. Psychiatry 2020, 52, 102066. [CrossRef] [PubMed]

4. Galli, F.; Pozzi, G.; Ruggiero, F.; Mameli, F.; Cavicchioli, M.; Barbieri, S.; Canevini, M.P.; Priori, A.; Pravettoni, G.; Sani, G.; et al. A Systematic Review and Provisional Metanalysis on Psychopathologic Burden on Health Care Workers of Coronavirus Outbreaks. Front. Psychiatry 2020, 11, 568664. [CrossRef]

5. Cai, H.; Tu, B.; Ma, J.; Chen, L.; Fu, L.; Jiang, Y.; Zhuang, Q. Psychological Impact and Coping Strategies of Frontline Medical Staff in Hunan between January and March 2020 during the Outbreak of Coronavirus Disease 2019 (COVID-19) in Hubei, China. Med. Sci. Monit. 2020, 26, e924171. [CrossRef]

6. Şahin, C.U.; Kulakaç, N. Exploring Anxiety Levels in Healthcare Workers during COVID-19 Pandemic: Turkey Sample. Curr. Psychol. 2021, 1-8. [CrossRef]

7. Liu, C.-Y.; Yang, Y.-Z.; Zhang, X.-M.; Xu, X.; Dou, Q.-L.; Zhang, W.-W.; Cheng, A.S.K. The Prevalence and Influencing Factors in Anxiety in Medical Workers Fighting COVID-19 in China: A Cross-Sectional Survey. Epidemiol. Infect. 2020, 148, e98. [CrossRef]

8. Barello, S.; Palamenghi, L.; Graffigna, G. Burnout and Somatic Symptoms among Frontline Healthcare Professionals at the Peak of the Italian COVID-19 Pandemic. Psychiatry Res. 2020, 290, 113129. [CrossRef]

9. Kotera, Y.; Ozaki, A.; Miyatake, H.; Tsunetoshi, C.; Nishikawa, Y.; Tanimoto, T. Mental Health of Medical Workers in Japan during COVID-19: Relationships with Loneliness, Hope and Self-Compassion. Curr. Psychol. 2021, 40, 6271-6274. [CrossRef]

10. Freudenberger, H.J. Staff Burn-Out. J. Soc. Issues 1974, 30, 159-165. [CrossRef]

11. World Health Organization CIE-11. Available online: https://icd.who.int/es (accessed on 7 January 2022). 
12. Burton, A.; Burgess, C.; Dean, S.; Koutsopoulou, G.Z.; Hugh-Jones, S. How Effective Are Mindfulness-Based Interventions for Reducing Stress among Healthcare Professionals? A Systematic Review and Meta-Analysis. Stress Health J. Int. Soc. Investig. Stress 2017, 33, 3-13. [CrossRef] [PubMed]

13. Bride, B.E.; Radey, M.; Figley, C.R. Measuring Compassion Fatigue. Clin. Soc. Work J. 2007, 35, 155-163. [CrossRef]

14. Figley, C.R. Compassion Fatigue: Coping with Secondary Traumatic Stress Disorder in Those Who Treat the Traumatized; Routledge: New York, NY, USA, 1995; ISBN 978-0-203-77738-1.

15. Galiana, L.; Sansó, N.; Muñoz-Martínez, I.; Vidal-Blanco, G.; Oliver, A.; Larkin, P.J. Palliative Care Professionals' Inner Life: Exploring the Mediating Role of Self-Compassion in the Prediction of Compassion Satisfaction, Compassion Fatigue, Burnout and Wellbeing. J. Pain Symptom Manag. 2021, 63, 112-123. [CrossRef]

16. Sansó, N.; Galiana, L.; Oliver, A.; Pascual, A.; Sinclair, S.; Benito, E. Palliative Care Professionals' Inner Life: Exploring the Relationships among Awareness, Self-Care, and Compassion Satisfaction and Fatigue, Burnout, and Coping with Death. J. Pain Symptom Manag. 2015, 50, 200-207. [CrossRef] [PubMed]

17. Arpacioglu, S.; Gurler, M.; Cakiroglu, S. Secondary Traumatization Outcomes and Associated Factors among the Health Care Workers Exposed to the COVID-19. Int. J. Soc. Psychiatry 2021, 67, 84-89. [CrossRef] [PubMed]

18. Shahrour, G.; Dardas, L.A. Acute Stress Disorder, Coping Self-Efficacy and Subsequent Psychological Distress among Nurses amid COVID-19. J. Nurs. Manag. 2020, 28, 1686-1695. [CrossRef] [PubMed]

19. Figley, C.R. Compassion Fatigue: Psychotherapists' Chronic Lack of Self Care. J. Clin. Psychol. 2002, 58, 1433-1441. [CrossRef]

20. Alharbi, J.; Jackson, D.; Usher, K. Compassion Fatigue in Critical Care Nurses. An Integrative Review of the Literature. Saudi Med. J. 2019, 40, 1087-1097. [CrossRef]

21. Stamm, B.H. Helping the Helpers Helping the Helpers: Compassion Satisfaction and Compassion Fatigue in Self-Care, Management, and Policy of Suicide Prevention Hotlines. In Resources for Community Suicide Prevention; Idaho State University Article; Idaho State University: Pocatello, ID, USA, 2012.

22. Stamm, B.H. Professional Quality of Life Measure: Compassion, Satisfaction, and Fatigue Version 5 (ProQOL); Center for Victim Research: Washington, DC, USA, 2009.

23. Hooper, C.; Craig, J.; Janvrin, D.R.; Wetsel, M.A.; Reimels, E. Compassion Satisfaction, Burnout, and Compassion Fatigue among Emergency Nurses Compared with Nurses in Other Selected Inpatient Specialties. J. Emerg. Nurs. 2010, 36, 420-427. [CrossRef]

24. Koh, M.Y.H.; Chong, P.H.; Neo, P.S.H.; Ong, Y.J.; Yong, W.C.; Ong, W.Y.; Shen, M.L.J.; Hum, A.Y.M. Burnout, Psychological Morbidity and Use of Coping Mechanisms among Palliative Care Practitioners: A Multi-Centre Cross-Sectional Study. Palliat. Med. 2015, 29, 633-642. [CrossRef]

25. Lizano, E.L. Examining the Impact of Job Burnout on the Health and Well-Being of Human Service Workers: A Systematic Review and Synthesis. Hum. Serv. Organ. Manag. Leadersh. Gov. 2015, 39, 167-181. [CrossRef]

26. Sansó, N.; Galiana, L.; Oliver, A.; Tomás-Salvá, M.; Vidal-Blanco, G. Predicting Professional Quality of Life and Life Satisfaction in Spanish Nurses: A Cross-Sectional Study. Int. J. Environ. Res. Public Health 2020, 17, 4366. [CrossRef] [PubMed]

27. Senturk, J.C.; Melnitchouk, N. Surgeon Burnout: Defining, Identifying, and Addressing the New Reality. Clin. Colon Rectal Surg. 2019, 32, 407-414. [CrossRef] [PubMed]

28. Panagioti, M.; Geraghty, K.; Johnson, J.; Zhou, A.; Panagopoulou, E.; Chew-Graham, C.; Peters, D.; Hodkinson, A.; Riley, R.; Esmail, A. Association between Physician Burnout and Patient Safety, Professionalism, and Patient Satisfaction: A Systematic Review and Meta-Analysis. JAMA Intern. Med. 2018, 178, 1317-1331. [CrossRef] [PubMed]

29. García-Iglesias, J.J.; Gómez-Salgado, J.; Martín-Pereira, J.; Fagundo-Rivera, J.; Ayuso-Murillo, D.; Martínez-Riera, J.R.; Ruiz-Frutos, C. Impact of SARS-CoV-2 (COVID-19) on the mental health of healthcare professionals: A systematic review. Rev. Esp. Salud Publica 2020, 94, e202007088.

30. Serrano-Ripoll, M.J.; Meneses-Echavez, J.F.; Ricci-Cabello, I.; Fraile-Navarro, D.; Fiol-deRoque, M.A.; Pastor-Moreno, G.; Castro, A.; Ruiz-Pérez, I.; Zamanillo Campos, R.; Gonçalves-Bradley, D.C. Impact of Viral Epidemic Outbreaks on Mental Health of Healthcare Workers: A Rapid Systematic Review and Meta-Analysis. J. Affect. Disord. 2020, 277, 347-357. [CrossRef]

31. Amanullah, S.; Ramesh Shankar, R. The Impact of COVID-19 on Physician Burnout Globally: A Review. Healthcare 2020, 8, 421. [CrossRef]

32. Raudenská, J.; Steinerová, V.; Javůrková, A.; Urits, I.; Kaye, A.D.; Viswanath, O.; Varrassi, G. Occupational Burnout Syndrome and Post-Traumatic Stress among Healthcare Professionals during the Novel Coronavirus Disease 2019 (COVID-19) Pandemic. Best Pract. Res. Clin. Anaesthesiol. 2020, 34, 553-560. [CrossRef]

33. Restauri, N.; Sheridan, A.D. Burnout and Posttraumatic Stress Disorder in the Coronavirus Disease 2019 (COVID-19) Pandemic: Intersection, Impact, and Interventions. J. Am. Coll. Radiol. 2020, 17, 921-926. [CrossRef]

34. Sharifi, M.; Asadi-Pooya, A.A.; Mousavi-Roknabadi, R.S. Burnout among Healthcare Providers of COVID-19; A Systematic Review of Epidemiology and Recommendations. Arch. Acad. Emerg. Med. 2021, 9, e7. [CrossRef]

35. Benfante, A.; Di Tella, M.; Romeo, A.; Castelli, L. Traumatic Stress in Healthcare Workers during COVID-19 Pandemic: A Review of the Immediate Impact. Front. Psychol. 2020, 11, 569935. [CrossRef] [PubMed]

36. Moher, D.; Shamseer, L.; Clarke, M.; Ghersi, D.; Liberati, A.; Petticrew, M.; Shekelle, P.; Stewart, L.A.; PRISMA-P Group. Preferred Reporting Items for Systematic Review and Meta-Analysis Protocols (PRISMA-P) 2015 Statement. Syst. Rev. 2015, 4, 1-9. [CrossRef] [PubMed] 
37. Aafjes-van Doorn, K.; Békés, V.; Prout, T.A.; Hoffman, L. Psychotherapists' Vicarious Traumatization during the COVID-19 Pandemic. Psychol. Trauma Theory Res. Pract. Policy 2020, 12, S148-S150. [CrossRef] [PubMed]

38. Abdelhafiz, A.S.; Ali, A.; Ziady, H.H.; Maaly, A.M.; Alorabi, M.; Sultan, E.A. Prevalence, Associated Factors, and Consequences of Burnout among Egyptian Physicians during COVID-19 Pandemic. Front. Public Health 2020, 8, 864. [CrossRef]

39. Aebischer, O.; Weilenmann, S.; Gachoud, D.; Méan, M.; Spiller, T.R. Physical and Psychological Health of Medical Students Involved in the Coronavirus Disease 2019 Response in Switzerland. Swiss. Med. Wkly. 2020, 150, w20418. [CrossRef]

40. Jha, S.S.; Shah, S.; Calderon, M.D.; Soin, A.; Manchikanti, L. The Effect of COVID-19 on Interventional Pain Management Practices: A Physician Burnout Survey. Pain Physician 2020, 23, S271-S282.

41. Azoulay, E.; De Waele, J.; Ferrer, R.; Staudinger, T.; Borkowska, M.; Povoa, P.; Iliopoulou, K.; Artigas, A.; Schaller, S.J.; Hari, M.S.; et al. Symptoms of Burnout in Intensive Care Unit Specialists Facing the COVID-19 Outbreak. Ann. Intensive Care 2020, 10, 110. [CrossRef]

42. Barello, S.; Palamenghi, L.; Graffigna, G. Stressors and Resources for Healthcare Professionals during the COVID-19 Pandemic: Lesson Learned From Italy. Front. Psychol. 2020, 11, 2179. [CrossRef]

43. Blanco-Donoso, L.M.; Moreno-Jiménez, J.; Amutio, A.; Gallego-Alberto, L.; Moreno-Jiménez, B.; Garrosa, E. Stressors, Job Resources, Fear of Contagion, and Secondary Traumatic Stress among Nursing Home Workers in Face of the COVID-19: The Case of Spain. J. Appl. Gerontol. 2021, 40, 244-256. [CrossRef]

44. Buselli, R.; Corsi, M.; Baldanzi, S.; Chiumiento, M.; Del Lupo, E.; Dell'Oste, V.; Bertelloni, C.A.; Massimetti, G.; Dell'Osso, L.; Cristaudo, A.; et al. Professional Quality of Life and Mental Health Outcomes among Health Care Workers Exposed to Sars-Cov-2 (COVID-19). Int. J. Environ. Res. Public Health 2020, 17, 6180. [CrossRef]

45. Çelmeçe, N.; Menekay, M. The Effect of Stress, Anxiety and Burnout Levels of Healthcare Professionals Caring for COVID-19 Patients on Their Quality of Life. Front. Psychol. 2020, 11, 3329. [CrossRef] [PubMed]

46. Chen, J.; Liu, X.; Wang, D.; Jin, Y.; He, M.; Ma, Y.; Zhao, X.; Song, S.; Zhang, L.; Xiang, X.; et al. Risk Factors for Depression and Anxiety in Healthcare Workers Deployed during the COVID-19 Outbreak in China. Soc. Psychiatry Psychiatr. Epidemiol. 2021, 56, 47-55. [CrossRef] [PubMed]

47. Chen, R.; Sun, C.; Chen, J.-J.; Jen, H.-J.; Kang, X.L.; Kao, C.-C.; Chou, K.-R. A Large-Scale Survey on Trauma, Burnout, and Posttraumatic Growth among Nurses during the COVID-19 Pandemic. Int. J. Ment. Health Nurs. 2021, 30, 102-116. [CrossRef]

48. Chor, W.P.D.; Ng, W.M.; Cheng, L.; Situ, W.; Chong, J.W.; Ng, L.Y.A.; Mok, P.L.; Yau, Y.W.; Lin, Z. Burnout amongst Emergency Healthcare Workers during the COVID-19 Pandemic: A Multi-Center Study. Am. J. Emerg. Med. 2021, 46, 700-702. [CrossRef]

49. Civantos, A.M.; Bertelli, A.; Gonçalves, A.; Getzen, E.; Chang, C.; Long, Q.; Rajasekaran, K. Mental Health among Head and Neck Surgeons in Brazil during the COVID-19 Pandemic: A National Study. Am. J. Otolaryngol. 2020, 41, 102694. [CrossRef] [PubMed]

50. Civantos, A.M.; Byrnes, Y.; Chang, C.; Prasad, A.; Chorath, K.; Poonia, S.K.; Jenks, C.M.; Bur, A.M.; Thakkar, P.; Graboyes, E.M.; et al. Mental Health among Otolaryngology Resident and Attending Physicians during the COVID-19 Pandemic: National Study. Head Neck 2020, 42, 1597-1609. [CrossRef] [PubMed]

51. Coleman, J.R.; Abdelsattar, J.M.; Glocker, R.J.; Carmichael, H.; Vigneshwar, N.G.; Ryan, R.; Qiu, Q.; Nayyar, A.; Visenio, M.R.; Sonntag, C.C.; et al. COVID-19 Pandemic and the Lived Experience of Surgical Residents, Fellows, and Early-Career Surgeons in the American College of Surgeons. J. Am. Coll. Surg. 2021, 232, 119-135. [CrossRef]

52. Correia, I.; Almeida, A.E. Organizational Justice, Professional Identification, Empathy, and Meaningful Work during COVID-19 Pandemic: Are They Burnout Protectors in Physicians and Nurses? Front. Psychol. 2020, 11, 3545. [CrossRef]

53. Cravero, A.L.; Kim, N.J.; Feld, L.D.; Berry, K.; Rabiee, A.; Bazarbashi, N.; Bassin, S.; Lee, T.-H.; Moon, A.M.; Qi, X.; et al. Impact of Exposure to Patients with COVID-19 on Residents and Fellows: An International Survey of 1420 Trainees. Postgrad. Med. J. 2021, 97, 706-715. [CrossRef]

54. Demartini, B.; Nisticò, V.; D’Agostino, A.; Priori, A.; Gambini, O. Early Psychiatric Impact of COVID-19 Pandemic on the General Population and Healthcare Workers in Italy: A Preliminary Study. Front. Psychiatry 2020, 11, 1475. [CrossRef]

55. de Wit, K.; Mercuri, M.; Wallner, C.; Clayton, N.; Archambault, P.; Ritchie, K.; Gérin-Lajoie, C.; Gray, S.; Schwartz, L.; Chan, T.; et al. Canadian Emergency Physician Psychological Distress and Burnout during the First 10 Weeks of COVID-19: A Mixed-Methods Study. J. Am. Coll. Emerg. Physicians Open 2020, 1, 1030-1038. [CrossRef] [PubMed]

56. Di Monte, C.; Monaco, S.; Mariani, R.; Di Trani, M. From Resilience to Burnout: Psychological Features of Italian General Practitioners during COVID-19 Emergency. Front. Psychol. 2020, 11, 2476. [CrossRef] [PubMed]

57. Dimitriu, M.C.T.; Pantea-Stoian, A.; Smaranda, A.C.; Nica, A.A.; Carap, A.C.; Constantin, V.D.; Davitoiu, A.M.; Cirstoveanu, C.; Bacalbasa, N.; Bratu, O.G.; et al. Burnout Syndrome in Romanian Medical Residents in Time of the COVID-19 Pandemic. Med. Hypotheses 2020, 144, 109972. [CrossRef]

58. Dincer, B.; Inangil, D. The Effect of Emotional Freedom Techniques on Nurses' Stress, Anxiety, and Burnout Levels during the COVID-19 Pandemic: A Randomized Controlled Trial. Explore 2021, 17, 109-114. [CrossRef]

59. Dinibutun, S.R. Factors Associated with Burnout among Physicians: An Evaluation during a Period of COVID-19 Pandemic. J. Healthc. Leadersh. 2020, 12, 85-94. [CrossRef] [PubMed]

60. Dosil, M.; Ozamiz-Etxebarria, N.; Redondo, I.; Picaza, M.; Jaureguizar, J. Psychological Symptoms in Health Professionals in Spain after the First Wave of the COVID-19 Pandemic. Front. Psychol. 2020, 11, 606121. [CrossRef]

61. Duarte, I.; Teixeira, A.; Castro, L.; Marina, S.; Ribeiro, C.; Jácome, C.; Martins, V.; Ribeiro-Vaz, I.; Pinheiro, H.C.; Silva, A.R.; et al. Burnout among Portuguese Healthcare Workers during the COVID-19 Pandemic. BMC Public Health 2020, 20, 1885. [CrossRef] 
62. Elhadi, M.; Msherghi, A.; Elgzairi, M.; Alhashimi, A.; Bouhuwaish, A.; Biala, M.; Abuelmeda, S.; Khel, S.; Khaled, A.; Alsoufi, A.; et al. Burnout Syndrome among Hospital Healthcare Workers during the COVID-19 Pandemic and Civil War: A Cross-Sectional Study. Front. Psychiatry 2020, 11, 1426. [CrossRef]

63. El Haj, M.; Allain, P.; Annweiler, C.; Boutoleau-Bretonnière, C.; Chapelet, G.; Gallouj, K.; Kapogiannis, D.; Roche, J.; Boudoukha, A.H. Burnout of Healthcare Workers in Acute Care Geriatric Facilities during the COVID-19 Crisis: An Online-Based Study. J. Alzheimer's Dis. 2020, 78, 847-852. [CrossRef]

64. Franza, F.; Basta, R.; Pellegrino, F.; Solomita, B.; Fasano, V. The Role of Fatigue of Compassion, Burnout and Hopelessness in Healthcare: Experience in the Time of COVID-19 Outbreak. Psychiatr. Danub. 2020, 32, 10-14.

65. Franza, F.; Pellegrino, F.; Buono, G.D.; Solomita, B.; Fasano, V. Compassion Fatigue, Burnout and Hopelessness of the Health Workers in COVID-19 Pandemic Emergency. Eur. Neuropsychopharmacol. 2020, 40, S476-S477. [CrossRef]

66. Giusti, E.M.; Pedroli, E.; D’Aniello, G.E.; Stramba Badiale, C.; Pietrabissa, G.; Manna, C.; Stramba Badiale, M.; Riva, G.; Castelnuovo, G.; Molinari, E. The Psychological Impact of the COVID-19 Outbreak on Health Professionals: A Cross-Sectional Study. Front. Psychol. 2020, 11, 1684. [CrossRef] [PubMed]

67. Sarboozi Hoseinabadi, T.; Kakhki, S.; Teimori, G.; Nayyeri, S. Burnout and Its Influencing Factors between Frontline Nurses and Nurses from Other Wards during the Outbreak of Coronavirus Disease -COVID-19- in Iran. Investig. Educ. Enferm. 2020, $38,1-12$. [CrossRef]

68. Hu, D.; Kong, Y.; Li, W.; Han, Q.; Zhang, X.; Zhu, L.X.; Wan, S.W.; Liu, Z.; Shen, Q.; Yang, J.; et al. Frontline Nurses' Burnout, Anxiety, Depression, and Fear Statuses and Their Associated Factors during the COVID-19 Outbreak in Wuhan, China: A Large-Scale Cross-Sectional Study. EClinicalMedicine 2020, 24, 100424. [CrossRef]

69. Jose, S.; Dhandapani, M.; Cyriac, M.C. Burnout and Resilience among Frontline Nurses during COVID-19 Pandemic: A CrossSectional Study in the Emergency Department of a Tertiary Care Center, North India. Indian J. Crit. Care Med. 2020, 24, 1081-1088. [CrossRef]

70. Kannampallil, T.G.; Goss, C.W.; Evanoff, B.A.; Strickland, J.R.; McAlister, R.P.; Duncan, J. Exposure to COVID-19 Patients Increases Physician Trainee Stress and Burnout. PLoS ONE 2020, 15, e0237301. [CrossRef]

71. Kelker, H.; Yoder, K.; Musey, P.; Harris, M.; Johnson, O.; Sarmiento, E.; Vyas, P.; Henderson, B.; Adams, Z.; Welch, J.L. Longitudinal Prospective Study of Emergency Medicine Provider Wellness across Ten Academic and Community Hospitals during the Initial Surge of the COVID-19 Pandemic. Res. Sq. 2020. [CrossRef]

72. Khalafallah, A.M.; Lam, S.; Gami, A.; Dornbos, D.L.; Sivakumar, W.; Johnson, J.N.; Mukherjee, D. Burnout and Career Satisfaction among Attending Neurosurgeons during the COVID-19 Pandemic. Clin. Neurol. Neurosurg. 2020, 198, 106193. [CrossRef]

73. Khalafallah, A.M.; Lam, S.; Gami, A.; Dornbos, D.L.; Sivakumar, W.; Johnson, J.N.; Mukherjee, D. A National Survey on the Impact of the COVID-19 Pandemic upon Burnout and Career Satisfaction among Neurosurgery Residents. J. Clin. Neurosci. 2020, 80, 137-142. [CrossRef]

74. Khasne, R.W.; Dhakulkar, B.S.; Mahajan, H.C.; Kulkarni, A.P. Burnout among Healthcare Workers during COVID-19 Pandemic in India: Results of a Questionnaire-Based Survey. Indian J. Crit. Care Med. 2020, 24, 664-671. [CrossRef]

75. Khattak, S.R.; Saeed, I.; Rehman, S.U.; Fayaz, M. Impact of Fear of COVID-19 Pandemic on the Mental Health of Nurses in Pakistan. J. Loss Trauma 2021, 26, 421-435. [CrossRef]

76. Lázaro-Pérez, C.; Martínez-López, J.Á.; Gómez-Galán, J.; López-Meneses, E. Anxiety About the Risk of Death of Their Patients in Health Professionals in Spain: Analysis at the Peak of the COVID-19 Pandemic. Int. J. Environ. Res. Public Health 2020, 17, 5938. [CrossRef] [PubMed]

77. Lee, S.; Rozybakieva, Z.; Asimov, M.; Bagiyarova, F.; Tazhiyeva, A.; Ussebayeva, N.; Tanabayeva, S.; Fakhradiyev, I. Coping Strategy as a Way to Prevent Emotional Burnout in Primary Care Doctors: A Randomized Controlled Trial. Arch. Balk. Med. Union 2020, 55, 398-409. [CrossRef]

78. Li, Z.; Ge, J.; Yang, M.; Feng, J.; Qiao, M.; Jiang, R.; Bi, J.; Zhan, G.; Xu, X.; Wang, L.; et al. Vicarious Traumatization in the General Public, Members, and Non-Members of Medical Teams Aiding in COVID-19 Control. Brain. Behav. Immun. 2020, 88, 916-919. [CrossRef]

79. Litam, S.D.A.; Balkin, R.S. Moral Injury in Health-Care Workers during COVID-19 Pandemic. Traumatology 2021, 27, 14-19. [CrossRef]

80. Luceño-Moreno, L.; Talavera-Velasco, B.; García-Albuerne, Y.; Martín-García, J. Symptoms of Posttraumatic Stress, Anxiety, Depression, Levels of Resilience and Burnout in Spanish Health Personnel during the COVID-19 Pandemic. Int. J. Environ. Res. Public Health 2020, 17, 5514. [CrossRef]

81. Manzano García, G.; Ayala Calvo, J.C. The Threat of COVID-19 and Its Influence on Nursing Staff Burnout. J. Adv. Nurs. 2021, 77, 832-844. [CrossRef]

82. Martínez-López, J.Á.; Lázaro-Pérez, C.; Gómez-Galán, J.; Fernández-Martínez, M.D.M. Psychological Impact of COVID-19 Emergency on Health Professionals: Burnout Incidence at the Most Critical Period in Spain. J. Clin. Med. 2020, 9, 3029. [CrossRef]

83. Matsuo, T.; Kobayashi, D.; Taki, F.; Sakamoto, F.; Uehara, Y.; Mori, N.; Fukui, T. Prevalence of Health Care Worker Burnout during the Coronavirus Disease 2019 (COVID-19) Pandemic in Japan. JAMA Netw. Open 2020, 3, e2017271. [CrossRef]

84. Miller, A.G.; Roberts, K.J.; Hinkson, C.R.; Davis, G.; Strickland, S.L.; Rehder, K.J. Resilience and Burnout Resources in Respiratory Care Departments. Respir. Care 2021, 66, 715-723. [CrossRef] 
85. Murat, M.; Köse, S.; Savaşer, S. Determination of Stress, Depression and Burnout Levels of Front-Line Nurses during the COVID-19 Pandemic. Int. J. Ment. Health Nurs. 2021, 30, 533-543. [CrossRef] [PubMed]

86. Ng, K.Y.Y.; Zhou, S.; Tan, S.H.; Ishak, N.D.B.; Goh, Z.Z.S.; Chua, Z.Y.; Chia, J.M.X.; Chew, E.L.; Shwe, T.; Mok, J.K.Y.; et al. Understanding the Psychological Impact of COVID-19 Pandemic on Patients with Cancer, Their Caregivers, and Health Care Workers in Singapore. JCO Glob. Oncol. 2020, 6, 1494-1509. [CrossRef] [PubMed]

87. Osama, M.; Zaheer, F.; Saeed, H.; Anees, K.; Jawed, Q.; Syed, S.H.; Sheikh, B.A. Impact of COVID-19 on Surgical Residency Programs in Pakistan; A Residents' Perspective. Do Programs Need Formal Restructuring to Adjust with the "New Normal"? A Cross-Sectional Survey Study. Int. J. Surg. 2020, 79, 252-256. [CrossRef] [PubMed]

88. Park, S.Y.; Kim, B.; Jung, D.S.; Jung, S.I.; Oh, W.S.; Kim, S.-W.; Peck, K.R.; Chang, H.-H. The Korean Society of Infectious Diseases Psychological Distress among Infectious Disease Physicians during the Response to the COVID-19 Outbreak in the Republic of Korea. BMC Public Health 2020, 20, 1811. [CrossRef] [PubMed]

89. Pinho, A.S.; Morales, A.U.; Bernal, M.B.; Villarroel, P.E.V. Sintomatología asociada a trastornos de salud mental en trabajadores sanitarios en Paraguay: Efecto COVID-19. Rev. Interam. Psicol. Interam. J. Psychol. 2020, 54, 7.

90. Prasad, A.; Civantos, A.M.; Byrnes, Y.; Chorath, K.; Poonia, S.; Chang, C.; Graboyes, E.M.; Bur, A.M.; Thakkar, P.; Deng, J.; et al. Snapshot Impact of COVID-19 on Mental Wellness in Nonphysician Otolaryngology Health Care Workers: A National Study. OTO Open 2020, 4, 2473974X20948835. [CrossRef]

91. Rapisarda, F.; Vallarino, M.; Cavallini, E.; Barbato, A.; Brousseau-Paradis, C.; De Benedictis, L.; Lesage, A. The Early Impact of the COVID-19 Emergency on Mental Health Workers: A Survey in Lombardy, Italy. Int. J. Environ. Res. Public Health 2020, 17, 8615. [CrossRef]

92. Rodriguez, R.M.; Medak, A.J.; Baumann, B.M.; Lim, S.; Chinnock, B.; Frazier, R.; Cooper, R.J. Academic Emergency Medicine Physicians' Anxiety Levels, Stressors, and Potential Stress Mitigation Measures during the Acceleration Phase of the COVID-19 Pandemic. Acad. Emerg. Med. 2020, 27, 700-707. [CrossRef]

93. Ruiz-Fernández, M.D.; Ramos-Pichardo, J.D.; Ibáñez-Masero, O.; Cabrera-Troya, J.; Carmona-Rega, M.I.; Ortega-Galán, Á.M. Compassion Fatigue, Burnout, Compassion Satisfaction and Perceived Stress in Healthcare Professionals during the COVID-19 Health Crisis in Spain. J. Clin. Nurs. 2020, 29, 4321-4330. [CrossRef]

94. Aydin Sayilan, A.; Kulakaç, N.; Uzun, S. Burnout Levels and Sleep Quality of COVID-19 Heroes. Perspect. Psychiatr. Care 2021, 57, 1231-1236. [CrossRef]

95. Secosan, I.; Virga, D.; Crainiceanu, Z.P.; Bratu, T. The Mediating Role of Insomnia and Exhaustion in the Relationship between Secondary Traumatic Stress and Mental Health Complaints among Frontline Medical Staff during the COVID-19 Pandemic. Behav. Sci. 2020, 10, 164. [CrossRef] [PubMed]

96. Soto-Rubio, A.; Giménez-Espert, M.D.C.; Prado-Gascó, V. Effect of Emotional Intelligence and Psychosocial Risks on Burnout, Job Satisfaction, and Nurses' Health during the COVID-19 Pandemic. Int. J. Environ. Res. Public Health 2020, 17, 7998. [CrossRef] [PubMed]

97. Spiller, T.R.; Méan, M.; Ernst, J.; Sazpinar, O.; Gehrke, S.; Paolercio, F.; Petry, H.; Pfaltz, M.C.; Morina, N.; Aebischer, O.; et al. Development of Health Care Workers' Mental Health during the SARS-CoV-2 Pandemic in Switzerland: Two Cross-Sectional Studies. Psychol. Med. 2020, 1-4. [CrossRef] [PubMed]

98. Tan, B.Y.Q.; Kanneganti, A.; Lim, L.J.H.; Tan, M.; Chua, Y.X.; Tan, L.; Sia, C.H.; Denning, M.; Goh, E.T.; Purkayastha, S.; et al. Burnout and Associated Factors among Health Care Workers in Singapore during the COVID-19 Pandemic. J. Am. Med. Dir. Assoc. 2020, 21, 1751-1758.e5. [CrossRef] [PubMed]

99. Trumello, C.; Bramanti, S.M.; Ballarotto, G.; Candelori, C.; Cerniglia, L.; Cimino, S.; Crudele, M.; Lombardi, L.; Pignataro, S.; Viceconti, M.L.; et al. Psychological Adjustment of Healthcare Workers in Italy during the COVID-19 Pandemic: Differences in Stress, Anxiety, Depression, Burnout, Secondary Trauma, and Compassion Satisfaction between Frontline and Non-Frontline Professionals. Int. J. Environ. Res. Public Health 2020, 17, 8358. [CrossRef] [PubMed]

100. Vagni, M.; Maiorano, T.; Giostra, V.; Pajardi, D. Coping With COVID-19: Emergency Stress, Secondary Trauma and Self-Efficacy in Healthcare and Emergency Workers in Italy. Front. Psychol. 2020, 11, 2294. [CrossRef] [PubMed]

101. Wahlster, S.; Sharma, M.; Lewis, A.K.; Patel, P.V.; Hartog, C.S.; Jannotta, G.; Blissitt, P.; Kross, E.K.; Kassebaum, N.J.; Greer, D.M.; et al. The Coronavirus Disease 2019 Pandemic's Effect on Critical Care Resources and Health-Care Providers: A Global Survey. Chest 2021, 159, 619-633. [CrossRef]

102. Wu, Y.; Wang, J.; Luo, C.; Hu, S.; Lin, X.; Anderson, A.E.; Bruera, E.; Yang, X.; Wei, S.; Qian, Y. A Comparison of Burnout Frequency among Oncology Physicians and Nurses Working on the Frontline and Usual Wards during the COVID-19 Epidemic in Wuhan, China. J. Pain Symptom Manag. 2020, 60, e60-e65. [CrossRef]

103. Yörük, S.; Güler, D. The Relationship between Psychological Resilience, Burnout, Stress, and Sociodemographic Factors with Depression in Nurses and Midwives during the COVID-19 Pandemic: A Cross-Sectional Study in Turkey. Perspect. Psychiatr. Care 2021, 57, 390-398. [CrossRef]

104. Zerbini, G.; Ebigbo, A.; Reicherts, P.; Kunz, M.; Messman, H. Psychosocial Burden of Healthcare Professionals in Times of COVID-19-A Survey Conducted at the University Hospital Augsburg. GMS Ger. Med. Sci. 2020, 18, Doc05. [CrossRef]

105. Zhang, Y.; Wang, C.; Pan, W.; Zheng, J.; Gao, J.; Huang, X.; Cai, S.; Zhai, Y.; Latour, J.M.; Zhu, C. Stress, Burnout, and Coping Strategies of Frontline Nurses during the COVID-19 Epidemic in Wuhan and Shanghai, China. Front. Psychiatry 2020, 11, 1154. [CrossRef] [PubMed] 
106. Zhizhong, W.; Koenig, H.G.; Yan, T.; Jing, W.; Mu, S.; Hongyu, L.; Guangtian, L. Psychometric Properties of the Moral Injury Symptom Scale among Chinese Health Professionals during the COVID-19 Pandemic. BMC Psychiatry 2020, 20, 556. [CrossRef] [PubMed]

107. Sagherian, K.; Steege, L.M.; Cobb, S.J.; Cho, H. Insomnia, Fatigue and Psychosocial Well-Being during COVID-19 Pandemic: A Cross-Sectional Survey of Hospital Nursing Staff in the United States. J. Clin. Nurs. 2020. [CrossRef] [PubMed]

108. Lange, M.; Joo, S.; Couette, P.-A.; de Jaegher, S.; Joly, F.; Humbert, X. Impact on Mental Health of the COVID-19 Outbreak among Community Pharmacists during the Sanitary Lockdown Period. Ann. Pharm. Fr. 2020, 78, 459-463. [CrossRef] [PubMed]

109. Dobson, H.; Malpas, C.B.; Burrell, A.J.; Gurvich, C.; Chen, L.; Kulkarni, J.; Winton-Brown, T. Burnout and Psychological Distress amongst Australian Healthcare Workers during the COVID-19 Pandemic. Australas. Psychiatry Bull. R. Aust. N. Z. Coll. Psychiatr. 2021, 29, 26-30. [CrossRef] [PubMed]

110. Chuang, C.-H.; Tseng, P.-C.; Lin, C.-Y.; Lin, K.-H.; Chen, Y.-Y. Burnout in the Intensive Care Unit Professionals: A Systematic Review. Medicine 2016, 95, e5629. [CrossRef] [PubMed]

111. Hall, L.H.; Johnson, J.; Watt, I.; Tsipa, A.; O'Connor, D.B. Healthcare Staff Wellbeing, Burnout, and Patient Safety: A Systematic Review. PLoS ONE 2016, 11, e0159015. [CrossRef] [PubMed]

112. van Mol, M.M.C.; Kompanje, E.J.O.; Benoit, D.D.; Bakker, J.; Nijkamp, M.D. The Prevalence of Compassion Fatigue and Burnout among Healthcare Professionals in Intensive Care Units: A Systematic Review. PLoS ONE 2015, 10, e0136955. [CrossRef]

113. Cavanagh, N.; Cockett, G.; Heinrich, C.; Doig, L.; Fiest, K.; Guichon, J.R.; Page, S.; Mitchell, I.; Doig, C.J. Compassion Fatigue in Healthcare Providers: A Systematic Review and Meta-Analysis. Nurs. Ethics 2020, 27, 639-665. [CrossRef]

114. Ortega-Campos, E.; Vargas-Román, K.; Velando-Soriano, A.; Suleiman-Martos, N.; Cañadas-de la Fuente, G.A.; Albendín-García, L.; Gómez-Urquiza, J.L. Compassion Fatigue, Compassion Satisfaction, and Burnout in Oncology Nurses: A Systematic Review and Meta-Analysis. Sustainability 2020, 12, 72. [CrossRef]

115. Gómez-Urquiza, J.L.; Aneas-López, A.B.; Fuente-Solana, E.I.; Albendín-García, L.; Díaz-Rodríguez, L.; Fuente, G.A. Prevalence, Risk Factors, and Levels of Burnout Among Oncology Nurses: A Systematic Review. Oncol. Nurs. Forum 2016, 43, E104-E120. [CrossRef] [PubMed]

116. Rotenstein, L.S.; Torre, M.; Ramos, M.A.; Rosales, R.C.; Guille, C.; Sen, S.; Mata, D.A. Prevalence of Burnout among Physicians: A Systematic Review. JAMA 2018, 320, 1131-1150. [CrossRef] [PubMed]

117. Xie, W.; Chen, L.; Feng, F.; Okoli, C.T.C.; Tang, P.; Zeng, L.; Jin, M.; Zhang, Y.; Wang, J. The Prevalence of Compassion Satisfaction and Compassion Fatigue among Nurses: A Systematic Review and Meta-Analysis. Int. J. Nurs. Stud. 2021, 120, 103973. [CrossRef] [PubMed]

118. Bria, M.; Băban, A.; Dumitrascu, D. Systematic Review of Burnout Risk Factors among European Healthcare Professionals. Cogn. Brain Behav. 2012, 6, 423-452.

119. Heath, C.; Sommerfield, A.; von Ungern-Sternberg, B.S. Resilience Strategies to Manage Psychological Distress among Healthcare Workers during the COVID-19 Pandemic: A Narrative Review. Anaesthesia 2020, 75, 1364-1371. [CrossRef]

120. Chew, Q.H.; Wei, K.C.; Vasoo, S.; Sim, K. Psychological and Coping Responses of Health Care Workers toward Emerging Infectious Disease Outbreaks: A Rapid Review and Practical Implications for the COVID-19 Pandemic. J. Clin. Psychiatry 2020, 81, 16119. [CrossRef] 